\title{
Genetic landscape of long noncoding RNA (IncRNAs) in glioblastoma: identification of complex IncRNA regulatory networks and clinically relevant IncRNAs in glioblastoma
}

\author{
Yashna Paul ${ }^{1, *}$, Sannu Thomas ${ }^{1, *}$, Vikas Patil ${ }^{1,5, *}{ }^{2}$, Naveen Kumar ${ }^{1}$, Baisakhi Mondal ${ }^{1}$, \\ Alangar S. Hegde ${ }^{2}$, Arimappamagan Arivazhagan ${ }^{3}$, Vani Santosh ${ }^{4}$, Kulandaivelu \\ Mahalingam $^{5}$ and Kumaravel Somasundaram ${ }^{1}$ \\ ${ }^{1}$ Department of Microbiology and Cell Biology, Indian Institute of Science, 560012 Bangalore, India \\ ${ }^{2}$ Sri Satya Sai Institute of Higher Medical Sciences, 560066 Bangalore, India \\ ${ }^{3}$ Department of Neurosurgery, National Institute of Mental Health and Neuro Sciences, 560029 Bangalore, India \\ ${ }^{4}$ Department of Neuropathology, National Institute of Mental Health and Neuro Sciences, 560029 Bangalore, India \\ ${ }^{5}$ Department of Bio-Medical Sciences, School of Biosciences and Technology, VIT University, 632014 Vellore, India \\ *These authors contributed equally to this work \\ Correspondence to: Kumaravel Somasundaram, email: ksomasundaram $1 @ g m a i l . c o m$ \\ Keywords: IncRNA; glioblastoma; ceRNA; ANRIL; CDKN2A-ASI \\ Received: November 26, $2017 \quad$ Accepted: April 20, $2018 \quad$ Published: July 03, 2018 \\ Copyright: Paul et al. This is an open-access article distributed under the terms of the Creative Commons Attribution License 3.0 \\ (CC BY 3.0), which permits unrestricted use, distribution, and reproduction in any medium, provided the original author and source \\ are credited.
}

\section{ABSTRACT}

The major part of the genome that was previously called junk DNA has been shown to be dynamically transcribed to produce non-coding RNAs. Among them, the long non-coding RNAs (IncRNA) play diverse roles in the cellular context and are therefore involved in various diseases like cancer. LncRNA transcript profiling of glioblastoma $(n=19)$ and control brain samples $(n=9)$ identified 2,774 and 5,016 IncRNAs to be upregulated and downregulated in GBMs respectively. Correlation analysis of differentially regulated IncRNAs with mRNA and IncRNA identified several IncRNAs that may potentially regulate many tumor relevant mRNAs and IncRNAs both at nearby locations (cis) and far locations (trans). Integration of our data set with TCGA GBM RNA-Seq data $(n=172)$ revealed many IncRNAs as a host as well as decoy for many tumor regulated miRNAs. The expression pattern of seven IncRNAs- HOXD-AS2, RP4-792G4.2, CRNDE, ANRIL, RP11-389G6.3, RP11-325122.2 and AC123886.2 was validated by TCGA RNA-Seq data and RT-qPCR. Silencing ANRIL, a GBM upregulated IncRNA, inhibited glioma cell proliferation and colony growth. Cox regression analysis identified several prognostic IncRNAs. An IncRNA risk score derived from five InRNAs-RP6-99M1.2, SOX21-AS1, CTD-2127H9.1, RP11-375B1.3 and RP3-449M8.9 predicted survival independent of all other markers. Multivariate cox regression analysis involving G-CIMP, IDH1 mutation, MGMT promoter methylation identified IncRNA risk score to be an independent poor predictor of GBM survival. The IncRNA risk score also stratified GBM patients into low and high risk with significant survival difference. Thus our study demonstrates the importance of IncRNA in GBM pathology and underscores the potential possibility of targeting IncRNA for GBM therapy. 


\section{INTRODUCTION}

The importance of non-coding RNAs in cellular functions has been widely reported and this in turn indicates their significance in various diseases like cancer. IncRNAs account for a majority of the non-coding transcriptome of the cell and they have been demonstrated to function at the level of both transcriptional and posttranscriptional gene regulation [1]. Ranging from roles in blocking the activity of tumour suppressor genes and inhibiting angiogenesis $[2,3]$ to functioning as tumour suppressors [4], IncRNAs have important implications in tumour formation and progression. IncRNAs have also been implicated in activating invasion and metastasis $[5,6]$, raising the stakes on the role of lncRNAs in cancer.

lncRNAs have been shown to interact with RNA, DNA and even proteins [1]. This may be facilitated by their secondary structure or through a sequence-dependent mode. This property aids in the regulatory role they play at the transcriptional and post-transcriptional level. At the transcriptional level, they may act as a molecular signal for transcriptional activity or may guide specific complexes to the site of transcription. The enrichment of RNA Polymerase II at enhancer elements has been shown to be dependent on the transcription of lncRNAs in breast and prostate cancers [7]. This has added merit to the evidence of RNA transcription at enhancer elements and confirmed the production of enhancer associated lncRNAs [8]. In addition, loss-of-function studies have indicated a decreased expression of target genes when their associated lncRNAs are degraded [9]. These reports warranted a study into the effects of the differentially expressed IncRNAs which we have carried out by checking their correlation with mRNAs that were differentially expressed, on a sample to sample basis.

The role of miRNAs in diverse cellular physiological processes and in the cancer scenario is a well-studied field [10]. Among their many roles, miRNAs have also been shown to regulate the expression levels of lncRNAs. A study by Braconi and group in 2011, demonstrated the role of miR-29 in the promoter methylation of MEG3 in hepatocellular carcinoma. The reverse also holds true, with reports of lncRNAs serving as competing endogenous RNAs (ceRNAs) to sequester miRNAs and in turn, affect their mRNA targets [11-13].

While several reports described a genome-wide comprehensive characterization of regulation of IncRNA in many cancers [14-16], there are only few reports of lncRNA role in glioma. Few studies in recent years have reported the role of IncRNAs in glioma development [6, 17, 18]. A recent study using TCGA RNA-Seq data reported the regulation and survival association of lncRNAs in glioma including low grades subtypes and GBMs [19]. Our study, mainly focussed on GBMs, is a microarray based profiling lncRNA and mRNAs simultaneously that enabled us to identify coregulating lncRNA-mRNA and lncRNA-lncRNA pairs. We also used TCGA RNA-Seq to identify many lncRNAs that serve as host as well as decoy for many tumor regulated miRNAs. Cox proportional regression analysis revealed the survival associated lncRNAs with an lncRNA risk-score developed using most significant lncRNA identified to be an independent predictor of survival in GBM

\section{RESULTS}

\section{Comprehensive genome analysis of IncRNAs in GBM}

In this study, we have carried out an integrated comprehensive analysis by integrating the expression profile of IncRNAs and mRNA in glioblastoma (GBM) derived from our cohort with lncRNA, miRNA and miRNA expression datasets from TCGA to understand the role of lncRNA mediated gene regulation at the transcriptional and post-transcriptional levels in GBM biology. A schematic diagram of the various types of analyses carried out in this study is shown (Figure 1). This analysis identified clinically relevant lncRNAs with respect to GBM pathobiology. We report here a comprehensive study reporting the microarray based expression profiling of IncRNAs that have been stringently and reliably annotated $(n=30,586)$ in GBM. A microarray that interrogates lncRNA $(n=30,586)$ and mRNA $(n=26,109)$ simultaneously from Arraystar Inc. was used in this study. Comparison of lncRNA expression profile between GBM $(n=19)$ and control brain samples $(n=9)$ identified differentially regulated lncRNAs in GBM. Then the differentially expressed lncRNAs were correlated with differentially regulated mRNAs and lncRNAs to identify the lncRNAs that could potentially regulate mRNAs and lncRNAs in GBM. Next, we investigated their possible post-transcriptional gene regulation through their effect on miRNAs. Coordinates of miRNAs were obtained from miRBase and were mapped to those of lncRNAs in order to derive the lncRNAs that were probable sources of miRNAs. Similarly, lncRNAs that are predicted to target mature miRNAs and thereby probably sequestering them were also derived. From this, lncRNA-miRNA-mRNA sponge modules were identified such that lncRNA could act as sponges in regulating mRNA expression positively by targeting miRNAs. TCGA RNA-Seq data was used to identify prognostic lncRNAs and lncRNA signature was found. The risk score predicted the IncRNA signature to be an independent poor prognostic indicator and it could divide GBMs into high and low risk patients. We have also validated the expression pattern of a number of IncRNAs by RT-qPCR. ANRIL, one of the GBM upregulated lncRNAs was taken up for in-depth functional investigation.

\section{IncRNAs are highly dysregulated in glioblastoma}

To identify the IncRNAs that are dysregulated in GBM, the lncRNA expression profile derived from 
control brain samples $(n=9)$ and GBM $(n=19)$ was compared (Supplementary Table 1; see methods for a more detailed description). Among the 30,586 lncRNAs transcripts profiled, lncRNAs with a difference of greater than absolute fold change $>1.5$ in GBM over the control brain samples and a $p$ value $<0.05$, were taken as significantly dysregulated. There were 2,774 lncRNA transcripts that were upregulated and 5,016 lncRNAs transcripts that were downregulated in GBM (Figure 2A; Supplementary Table 2). Next, we attempted to classify the dysregulated lncRNA into different lncRNA subtypes based on their position and direction of transcription in relation to overlapping or nearby mRNA genes (Figure 2B). The dysregulated lncRNA transcripts were classified into the following sub types: 'intergenic', when they lay in the interval between other protein coding genes; 'intronic antisense', when they were derived from the antisense to the intron of another gene; 'intron sense overlapping', when they were derived from intron of a gene; 'natural antisense', when the overlap was with exon(s) of a gene on the antisense strand; "exon sense overlapping', when they overlapped with the exon(s) of a gene; 'bidirectional', when they were transcribed to opposite direction to a protein coding gene with start points within 1000 bps. The contribution by each sub type of lncRNAs among dysregulated lncRNAs is shown (Figure 2C and Supplementary Figure 1A). The highest number of dysregulated lncRNAs fell into the intergenic group $(n=4,720 ; 60.59 \%)$, indicating that the majority of lncRNAs are regulated as independent transcription units. Next, we removed the exon sense overlapping lncRNAs from the differentially regulated lncRNA to get rid of the possibility of ambiguity that their probes in the microarray might measure both over lapping mRNA and lncRNA. The coordinates of exon sense overlapping lncRNAs that were overlapping entirely with that of the mRNAs were alone removed (Supplementary Figure 1B). This strategy would also aid in avoiding complications which could arise during attempts to validate these lncRNA transcripts in future studies. This step resulted in GBM upregulated

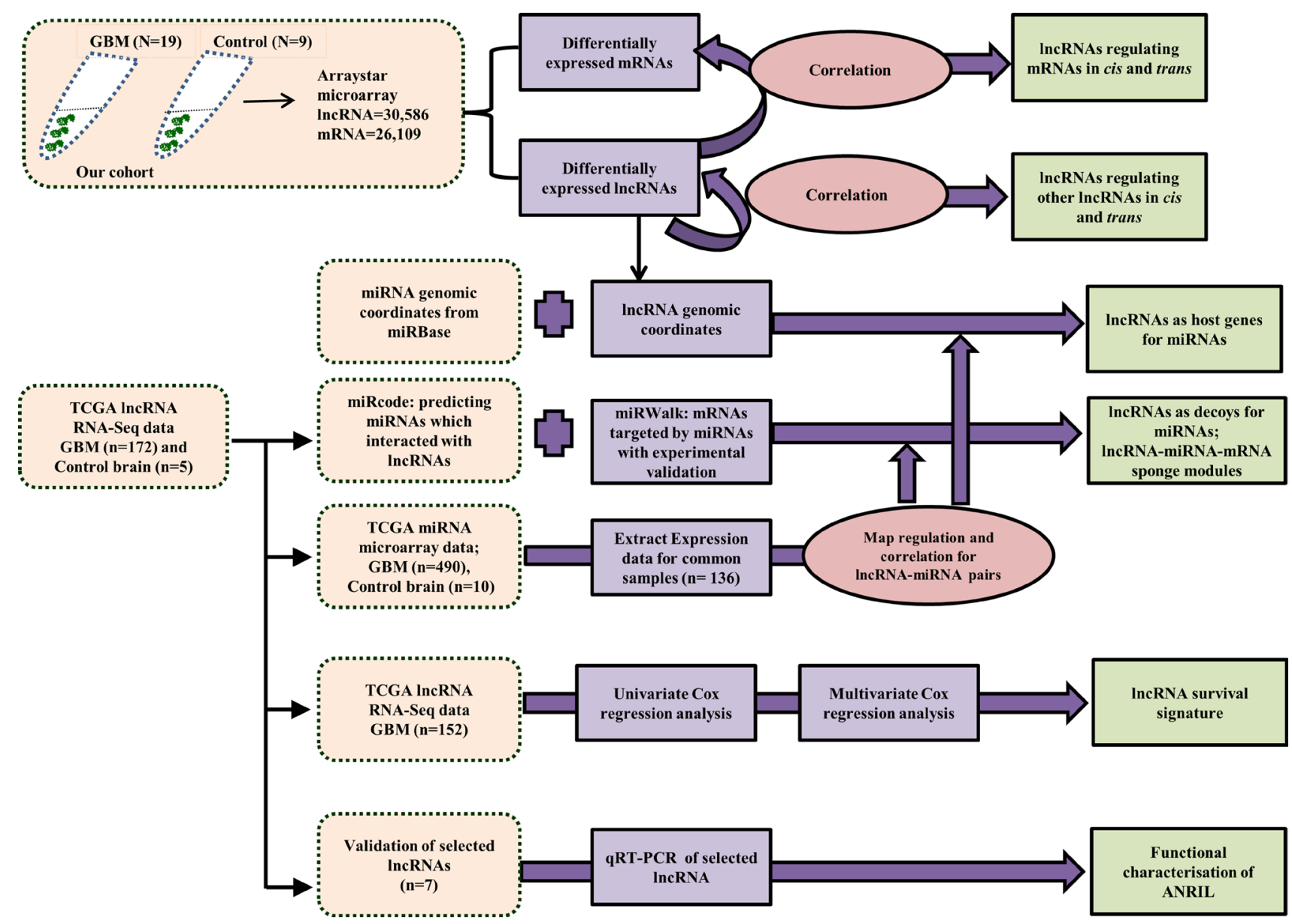

Figure 1: Scheme of the computational analyses carried out in the study. The data used for the analysis was either procured from our cohort (lncRNA \& mRNA microarray of 19 GBM and 9 control brains), from TCGA (lncRNA RNA-Seq data, miRNA microarray data) or from miRBase (miRNA genomic coordinates, miRNA sequences). The boxes in light pink indicate the source of the data used for the analysis, the purple boxes indicate the input data taken from the different sources, the dark pink boxes indicate the exercises carried out and the green boxes are indicative of the output obtained. 
and downregulated lncRNAs to 2268 transcripts (1838 lncRNAs) and 4595 transcripts (3938 lncRNAs) respectively (Figure 2D; Supplementary Table 2).

\section{Potential role of LncRNAs in the regulation of mRNA and IncRNA transcripts}

Correlations between lncRNAs and mRNAs based on their expression profiles have reportedly pointed at coregulation or functional relatedness [20]. Since the lncRNA microarray from Arraystar also profiled mRNA $(n=$ $26,109)$ expression, we were able to carry out a sample to sample correlation expression analysis between lncRNAs and mRNAs. mRNA transcriptome data analysis revealed that there were 2,806 mRNAs upregulated and 2,270 mRNAs downregulated with a difference of greater than absolute fold change greater than 2 and $p$ value $<0.05$ in
GBM over control brain samples (Supplementary Figure 2A; Supplementary Table 3). Supervised hierarchical clustering led to their partitioning of mRNA transcripts into distinct groups with the control brain samples and GBMs clustering separately (Supplementary Figure 2B). Cis regulation by lncRNAs has been demonstrated to occur over long genomic distances, as in the case of the lncRNA Air (IGF2AR) whose regulation spans over a distance of $300 \mathrm{~kb}$ [21]. Another study by Li et al. in 2013 also indicated the role of lncRNAs in stabilizing enhancer-promoter chromatin looping, thus explaining the distal regulation exhibited by cis-acting lncRNAs [22]. Based on the above cited studies, we took a genomic distance of $500 \mathrm{~kb}$ upstream and downstream of each of the lncRNA gene coordinate to identify $c i s$-regulated genes. The genes which are located beyond $\pm 500 \mathrm{~kb}$ upstream and downstream of the lncRNA gene were considered for trans-regulation by
$\mathbf{A}$

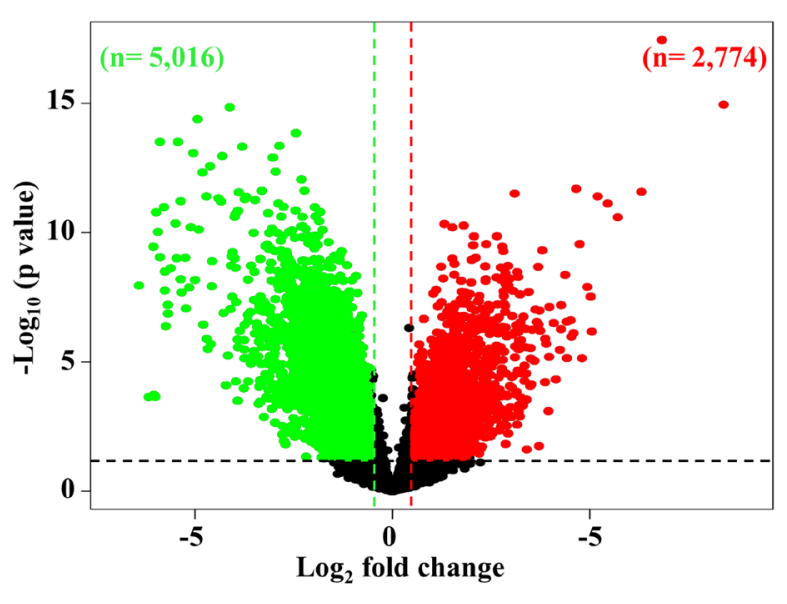

B

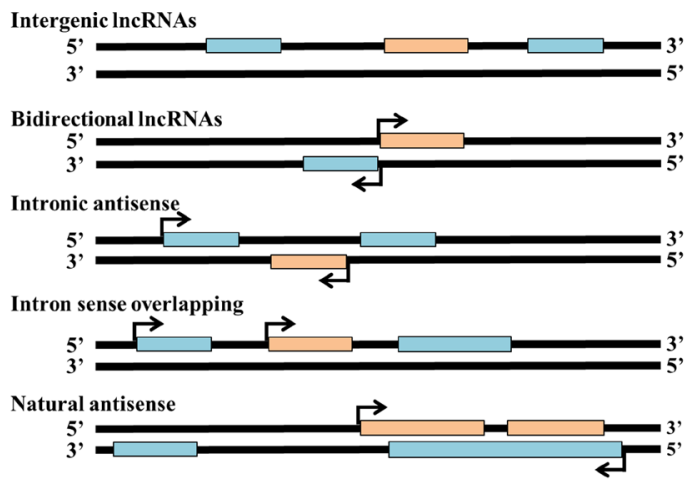

Exon sense overlapping

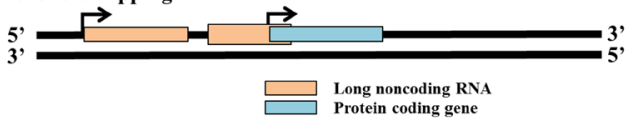

D

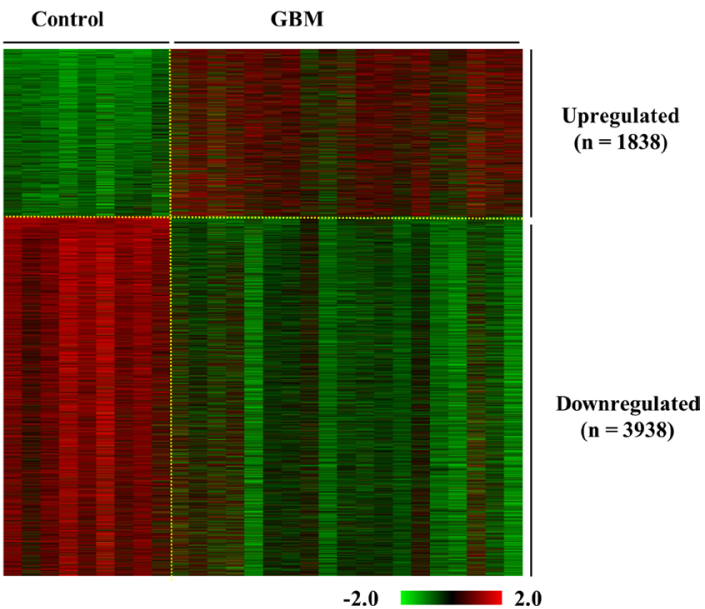

Figure 2: Differential regulation of IncRNAs in GBM. (A) Volcano plot indicating the upregulated (red), downregulated (green) and unregulated (black) lncRNAs in GBM as compared to control brain. 7,790 lncRNAs were differentially regulated at a difference of \pm 1.5 fold on the $\log _{2}$ scale over the control $(p<0.05)$, which included 2,774 transcripts that were upregulated and 5,016 that were downregulated. (B) Schematic representation of the different types of lncRNAs. The lncRNAs (denoted as pink boxes) are classified into 6 groups based on their relative gene positions with respect to nearby mRNA genes (denoted in blue). (C) Representation of the classification of dysregulated lncRNAs as a pie chart. The numbers of lncRNAs corresponding to each class are indicated. (D) Heat map showing the normalised $\log _{2}$ values of the dysregulated lncRNAs genes in GBM over the control samples. Upregulated genes are denoted in red and downregulated genes are shown in green. 
lncRNA. Then a spearman correlation analysis was carried out between differentially regulated lncRNAs (absolute fold change $>2$ and $p$ value $<0.05$ ) and differentially regulated mRNAs (absolute fold change $>2$ and $p$ value $<0.05$ ). The differentially regulated lncRNAs (absolute fold change $>2$ and $p$ value $<0.05$ ) were also correlated among themselves to identify the potential regulation of IncRNAs by other lncRNAs (Supplementary Figures 3 and 4). The correlation analysis between lncRNA and mRNA revealed many number of lncRNA-mRNA pairs that act on each other both cis- and trans fashion. There were 1148 cis acting lncRNA-mRNA transcript pairs with positive correlation among them (455 showed upregulation and 693 showed downregulation) and 848 lncRNA-mRNA transcript pairs that are negatively correlated, (165 lncRNA-mRNA pairs wherein upregulated lncRNAs potentially targeting downregulated mRNAs and 683 lncRNA-mRNA pairs wherein downregulated lncRNAs potentially targeting upregulated mRNAs) (Figure 3A; Supplementary Figures 3, 4). This analysis also identified 16,345 trans acting lncRNA-mRNA transcript pairs with positive correlation among them (2613 showed upregulation and 13732 showed downregulation) and 7114 lncRNAmRNA transcript pairs that are negatively correlated, (434 lncRNA-mRNA pairs wherein upregulated lncRNAs potentially targeting downregulated mRNAs and 6680 lncRNA-mRNA pairs wherein downregulated lncRNAs potentially targeting upregulated mRNAs) (Figure 3B; Supplementary Figure 3; Supplementary Table 5). Further to functional significance of regulated lncRNAs that were found to modulate mRNAs in glioma, the list of mRNAs (regulated by lncRNA as per Supplementary Tables 4 and 5) were subjected to pathway and gene ontology analysis by DAVID. As expected, this analysis showed enrichment of terms related to cell proliferation such as "cell cycle", "DNA replication", "DNA repair", "cell division" and pathways such as "Apoptosis" "p53 pathway, "ATM signalling” and "Telomere maintenance" (Supplementary Tables 6 and 7).

To identify lncRNAs that are regulated by other lncRNAs, a correlation analysis was carried out between differentially regulated lncRNAs (absolute fold change $>2$ and $p$ value $<0.05$ ). This analysis revealed many number of lncRNA-lncRNA pairs that act on each other both cis- and trans fashion. There were 1233 cis acting lncRNA-lncRNA transcript pairs with positive correlation among them (549 showed upregulation and 684 showed downregulation) and 149 lncRNA-lncRNA transcript pairs that are negatively correlated, (91 lncRNA-lncRNA pairs wherein upregulated lncRNAs potentially targeting downregulated lncRNAs and 58 lncRNA-lncRNA pairs wherein downregulated lncRNAs potentially targeting upregulated lncRNAs) (Figure 3C; Supplementary Figure 4; Supplementary Table 8). This analysis also identified 7651 trans acting lncRNA-lncRNA transcript pairs with positive correlation (359 showed upregulation and 7292 showed downregulation) and 493 lncRNA-lncRNA transcript pairs that are negatively correlated, (255
lncRNA-lncRNA pairs wherein upregulated lncRNAs potentially targeting downregulated lncRNAs and 238 lncRNA-lncRNA pairs wherein downregulated lncRNAs potentially targeting upregulated lncRNAs) (Figure 3D; Supplementary Figure 4; Supplementary Table 9).

\section{LncRNA regulation of miRNAs as host and sponge}

Next, we investigated the potential role of lncRNA in regulating miRNAs. miRNAs have been reported to be transcribed from the exons/introns of lncRNAs as well $[23,24]$, which adds another dimension to the regulation exhibited by lncRNAs at the post-transcriptional level. The expression of lncRNAs alters the levels of miRNAs, which in turn affect the expression of their target mRNAs [11]. We therefore checked for the presence of dysregulated lncRNAs that served as miRNA hosts. This analysis revealed that a total of 58 differentially regulated lncRNA carried 108 miRNA (see for detail methods; Supplementary Table 10). Further detailed analysis found that there are 13 lncRNA could potentially form the source of 24 miRNA as these IncRNA-miRNA pairs had similar regulation (Table 1). While ten IncRNA-miRNA pairs are found upregulated, three lncRNA-miRNA pairs are down regulated in GBM. There are three lncRNAs that carried more than one miRNA as host lncRNAs. While DLUE2 (Deleted In Lymphocytic Leukemia 2) carried two miRNAs- miR15A and miR16, LINC00478 lncRNA carried three miRNAs-let7c, miR99a and miR125b (Table 1; Supplementary Figure 5). It is interesting to note that the GBM upregulated IncRNA-MIR17HG carried miR17/92 cluster with six miRNAs- miR-17, miR-18a, miR-19a, miR-19b-1, miR-20a and miR-92a-1 (Table 1; Supplementary Figure 5).

lncRNAs have also been proposed to be more effective competing endogenous RNAs (ceRNA) as compared to mRNAs. We used an integrated analysis with multiple stringent steps involving GBM regulated lncRNAs, miRNAs and mRNAs through target prediction algorithms and experimentally validated miRNA-mRNA pairs to identify lncRNA-miRNA-mRNA sponge module networks (see for details methods). This analysis revealed that there are 409 IncRNA-miRNA-mRNA sponge modules (Supplementary Table 11) that are biologically relevant that the lncRNA in the module is highly likely to work as a sponge such that the target mRNA will be spared from inhibition by miRNAs. These sponge modules have the following characteristics: 1) all moleculeslncRNA, miRNA and mRNA are upregulated in GBM compared to control brain samples, 2) both IncRNA and mRNA have negative significant correlation with miRNA, 3) lncRNA and mRNA have significant positive correlation between them and 4) the abundance of InRNA transcripts was higher than that of mRNA in GBMs (Supplementary Table 11). Further, to find out important 
pathways that are regulated by these sponge modules, the unique set of mRNAs $(n=140)$ from this finally selected lncRNA-miRNA-mRNA sponge modules were used for DAVID pathway analysis (Supplementary Table 12). It is interesting to note that the terms related "Cell cycle" was identified by REACTOME, BIOCARTA and KEGG (Figure 4A). The cell cycle related genes E2F1, E2F2, CDKN1A, CDC25A, CDC25C and CDK6 expression appears to be regulated through several lncRNA sponges through the regulation of common miRNA(s) (Figure 4B-4E). Surprisingly, we also found CD28, IL10 and FAS related to activation of $\mathrm{T}$ and $\mathrm{B}$ lymphocytes, are upregulated and their high levels appears to be regulated by several sponge lncRNAs (Supplementary Figure 6A-6C). Thus, we have identified several lncRNA sponge molecules that appear play important role in regulating many mRNAs through their impact on miRNAs.

A

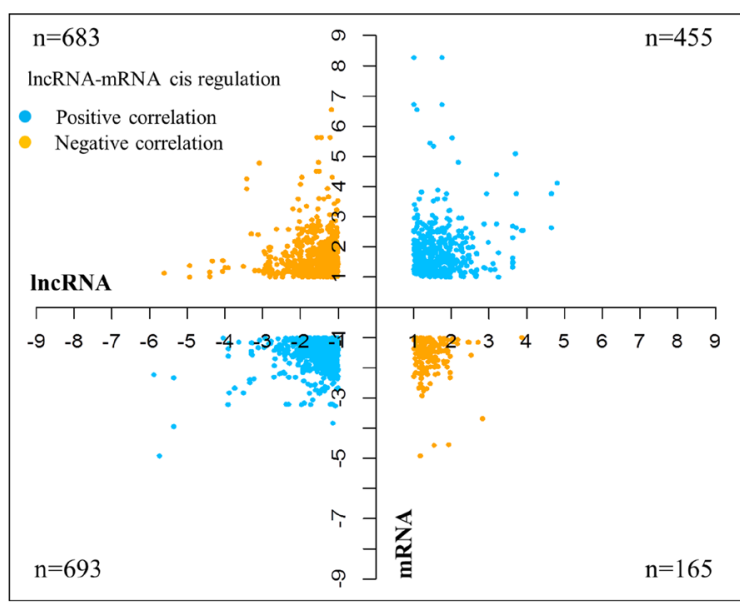

C

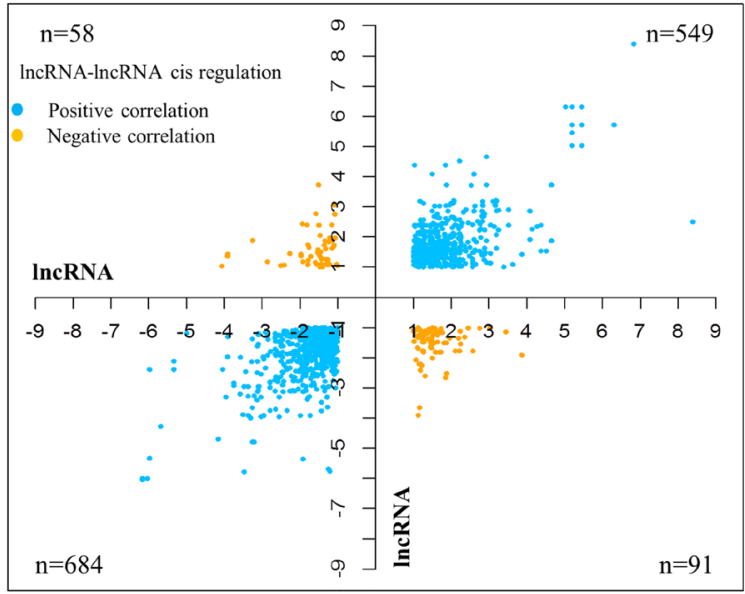

CDKN2B-AS1 or antisense noncoding RNA in the INK4 locus (ANRIL) is highly expressed in GBM but unlikely to be the repressor of INK4 locus

Next, we have taken seven differentially regulated lncRNAs for validation. Four upregulated lncRNAs (CDKN2B-AS2, HOXD-AS2, CRNDE and RP4-792G4.2) and three downregulated lncRNAs (RP11-389G6.3, RP11325122.2 and AC123886.2) as per microarray data also showed similar regulation in TCGA RNA-Seq data and RTqPCR data generated from the lab cohort (Figure 5A-5G). The lncRNA ANRIL (Antisense noncoding RNA in the INK4 locus), which is transcribed antisense to the INK4b-ARF-INK4a locus (Figure 6A), was considered for functional characterization with respect to glioma biology. We found the ANRIL transcript showed varying levels in glioma cell lines and silencing ANRIL in LN229

B

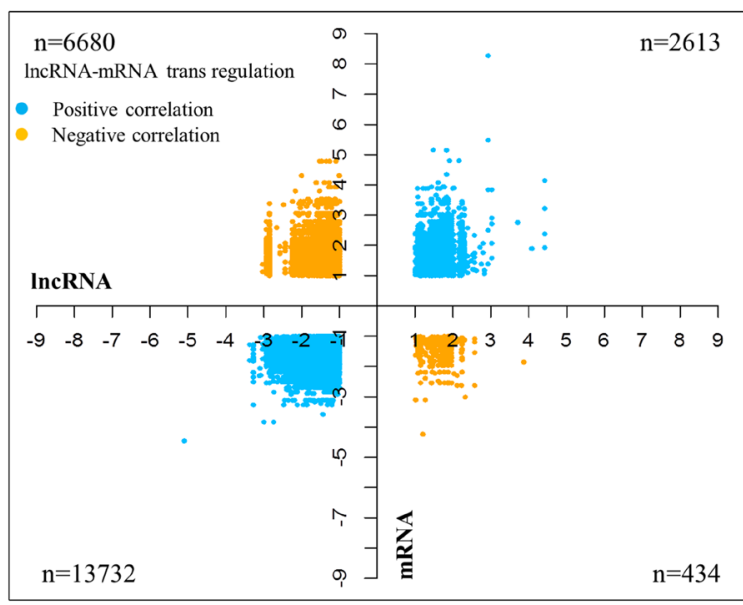

D

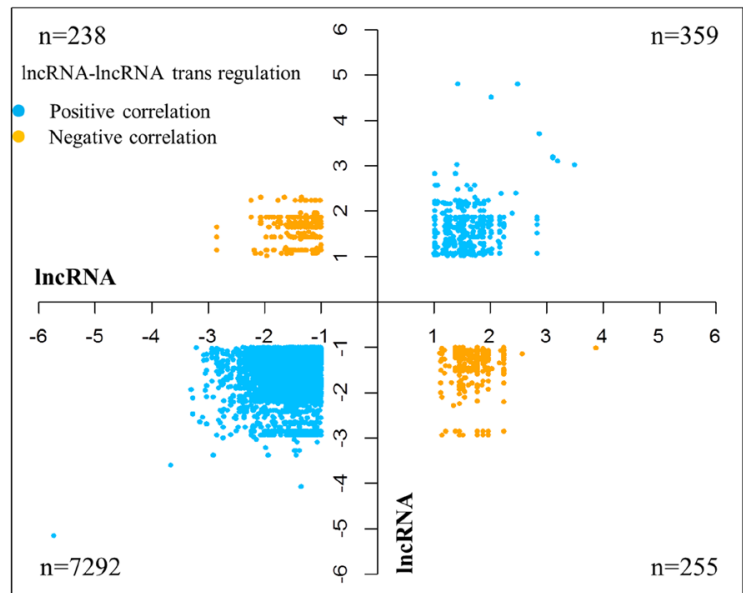

Figure 3: Correlation of IncRNAs with mRNAs and other IncRNA transcripts. Graphs showing the correlations between lncRNAs and mRNAs in cis (A) and trans (B). Each dot represents a lncRNA-mRNA correlating pair. The positive correlations are shown in blue and the yellow dots represent the negative correlations. LncRNA-lncRNA correlations are similarly depicted, with correlations in cis in graph (C) and those in trans in graph (D). Due to the massive number of correlation pairs obtained for mRNAs in trans, only the pairs having correlation coefficients of 0.9 and above have been represented in (B) and (D). The number of pairs for each of the correlations carried out $(n)$, are denoted in the graph. 
Table 1: LncRNA genes that behave as hosts or parent genes for miRNAs and miRNA families

\begin{tabular}{|c|c|c|c|c|c|c|c|c|c|}
\hline \multirow[t]{2}{*}{ Sr. No. } & \multirow[t]{2}{*}{ IncRNA name } & \multicolumn{3}{|c|}{$\begin{array}{c}\text { IncRNA TCGA RNA-seq } \\
\text { regulation }\end{array}$} & \multirow[t]{2}{*}{ miRNA name } & \multicolumn{2}{|c|}{$\begin{array}{c}\text { miRNA TCGA } \\
\text { microarray } \\
\text { regulation }\end{array}$} & \multicolumn{2}{|c|}{$\begin{array}{c}\text { TCGA sample wise } \\
\text { IncRNA-miRNA } \\
\text { correlation }\end{array}$} \\
\hline & & $\begin{array}{c}\log _{2} \text { Fold } \\
\text { Change }\end{array}$ & $p$ value & FDR & & $\begin{array}{c}\log _{2} \text { Fold } \\
\text { Change }\end{array}$ & $p$ value & $\begin{array}{c}\text { coefficient } \\
\text { (R) }\end{array}$ & $p$ value \\
\hline 1 & LINC00461 & 1.209 & 0.001 & 0.004 & hsa-miR-9-3p & 0.725 & $<0.0001$ & 0.380 & $<0.0001$ \\
\hline 2 & RMST & 1.814 & 0.004 & 0.017 & hsa-miR-135a-5p & 1.106 & $<0.0001$ & 0.414 & $<0.0001$ \\
\hline 3 & DNM3OS & 1.538 & 0.025 & 0.077 & hsa-miR-199a-3p & 0.923 & $<0.0001$ & 0.464 & $<0.0001$ \\
\hline 4 & LINC00472 & 0.837 & 0.020 & 0.065 & hsa-miR-30a-5p & 1.051 & $<0.0001$ & 0.477 & $<0.0001$ \\
\hline 5 & MIR155HG & 2.647 & $<0.0001$ & $<0.0001$ & hsa-miR-155-5p & 1.943 & $<0.0001$ & 0.554 & $<0.0001$ \\
\hline 6 & $\begin{array}{l}\text { BX537900/ } \\
\text { MIR124-2HG/ } \\
\text { LINC00966 }\end{array}$ & -3.348 & $<0.0001$ & $<0.0001$ & hsa-miR-124-3p & -5.962 & $<0.0001$ & 0.651 & $<0.0001$ \\
\hline 7 & $\begin{array}{l}\text { LOC100130155/ } \\
\text { MIR124-2HG/ } \\
\text { LINC0096 }\end{array}$ & -3.348 & $<0.0001$ & $<0.0001$ & hsa-miR-124-3p & -5.962 & $<0.0001$ & 0.651 & $<0.0001$ \\
\hline 8 & MIR210HG & 1.907 & $<0.0001$ & 0.001 & hsa-miR-210-3p & 2.170 & $<0.0001$ & 0.712 & $<0.0001$ \\
\hline 9 & MIR7-3HG & -3.299 & $<0.0001$ & $<0.0001$ & hsa-miR-7-5p & -3.734 & $<0.0001$ & 0.736 & $<0.0001$ \\
\hline \multirow[t]{8}{*}{10} & MIR17HG & 1.277 & 0.009 & 0.033 & hsa-miR-92a-3p & 1.206 & $<0.0001$ & 0.231 & 0.01 \\
\hline & & & & & hsa-miR-17-3p & 0.811 & $<0.0001$ & 0.127 & 0.169 \\
\hline & & & & & hsa-miR-17-5p & 1.137 & $<0.0001$ & 0.062 & 0.4990 \\
\hline & & & & & hsa-miR-18a-3p & 0.087 & $<0.0001$ & 0.304 & 0.0008 \\
\hline & & & & & hsa-miR-18a-5p & 0.774 & $<0.0001$ & 0.028 & 0.755 \\
\hline & & & & & hsa-miR-19a-3p & 1.031 & $<0.0001$ & 0.177 & 0.011 \\
\hline & & & & & hsa-miR-19b-3p & 1.063 & $<0.0001$ & 0.161 & 0.079 \\
\hline & & & & & hsa-miR-20a-5p & 1.3455 & $<0.0001$ & 0.063 & 0.492 \\
\hline \multirow[t]{2}{*}{11} & DLUE2 & 1.288 & $<0.0001$ & $<0.0001$ & hsa-miR-15a-5p & 1.466 & $<0.0001$ & 0.171 & 0.06 \\
\hline & & & & & hsa-miR-16-5p & 1.160 & $<0.0001$ & 0.113 & 0.22 \\
\hline \multirow[t]{3}{*}{13} & LINC00478 & 0.514 & 0.103 & 0.229 & hsa-let-7c-5p & 0.314 & $<0.0001$ & 0.420 & $<0.0001$ \\
\hline & & & & & hsa-miR-99a-5p & 0.786 & $<0.0001$ & 0.503 & $<0.0001$ \\
\hline & & & & & hsa-miR-125b-5p & -0.133 & 0.0644 & 0.29 & 0.001 \\
\hline
\end{tabular}

and T98G glioma cell lines inhibited cell proliferation and colony formation (Figure 6B-6H). INK4b/ARF/INK4a locus encodes two cyclin-dependent kinase inhibitors CDKN2B (p15) and CDKN2A (p16) as well as p19ARF which binds to MDM2 and promotes its degradation resulting in the p53 activation [25]. While the INK4b/ $\mathrm{ARF} / \mathrm{INK} 4 \mathrm{a}$ locus is shown to undergo deletion in GBM [26], epigenetic transcriptional repression of the locus by a repressive complex containing ANRIL and CBX7 has also been shown in prostate cancer [27]. We found ANRIL transcripts to be upregulated in GBM (Supplementary Figure 7A). Next, when we analysed the GBM samples divided into two groups on the basis of the presence of homozygous deletion in INK4b/ARF/INK4a locus, we found some interesting results. In contrast to entire cohort of GBM samples, we found a near significant or significant downregulation of ANRIL, CDKN2A and CDKN2B in GBMs with homozygous INK4a/INK4b co-deletion compared to that of control brain samples (Supplementary Figure 7A-7C). We also found a positive significant correlation between transcript levels of ANRIL and CDKN2A as well as CDKN2B in GBMs with homozygous deletion of the INK4b/ARF/INK4a locus (Supplementary Figure $7 \mathrm{E}$ and $7 \mathrm{~F}$ ). These results signify co-deletion of all three genes in GBMs with homozygous deletion of the INK4b/ARF/INK4a locus. The analysis of the GBM sub group with no copy number alterations revealed that the upregulation of ANRIL is largely restricted to GBMs with no copy number alterations in INK4b/ARF/INK4a locus (Supplementary Figure 7A). We also found a significant 
positive correlation between ANRIL and CDKN2A as well as CDKN2B in GBMs with no copy number aberrations (Supplementary Figure $7 \mathrm{G}$ and $7 \mathrm{H}$ ). Moreover, CBX7 is down regulated in GBMs regardless of the deletion status of INK4b/ARF/INK4a locus (Supplementary Figure 7D). These results demonstrate that INK4b/ARF/INK4a locus is not repressed by ANRIL in GBMs possibly due to the downregulation of CBX7. Thus, we conclude that ANRIL is upregulated in GBM and is an oncogenic lnRNA in GBM. Further, unlike what is shown in prostate cancer [27], ANRIL is not likely to be the repressor of INK4b/ ARF/INK4a locus in GBM.

\section{LncRNA expression correlates with survival of GBM patients}

To identify the IncRNAs that correlate to GBM survival, TCGA RNA-Seq data was used $(n=152)$. We defined the lncRNAs based on the manually annotated
lncRNA genes from Gencode v19, which included 13,853 lncRNAs [28]. Additional filters were used to use the lncRNAs, wherein the quantitation is reliable (see detail in methods). A total of 4840 lncRNA corresponding to intergenic, intron sense overlapping and processed transcripts and lncRNAs having at least $1 \mathrm{read}$ in $\geq 30 \%$ of TCGA RNA-Seq samples were considered for univariate Cox regression analysis. Out of $4840 \operatorname{lncRNAs}, 309$ showed a significant correlation with survival $(p$ value $<0.05)$ (Supplementary Table 13). Using stringent criteria, the top five lncRNAs were chosen for further analysis. While SOX21-AS1 showed good prognosis, the other four lncRNAs- RP6-99M1.2, CTD-2127H9.1, RP11-375B1.3 and RP3-449M8.9 showed poor prognosis (Figure 7A). An lncRNA risk score was made combining the survival prediction capability of all five lncRNAs by using a risk score formula (see for details methods). The lncRNA risk score predicted survival significantly in a univariate analysis as well as a multivariate analysis involving age,

A

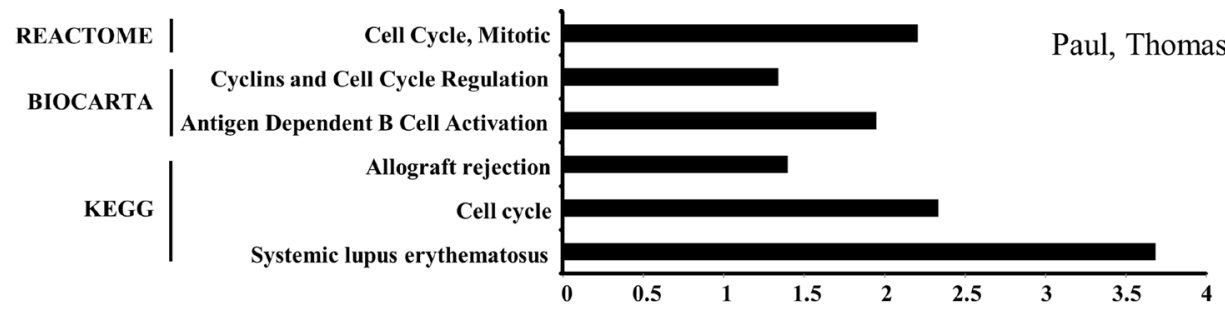

B

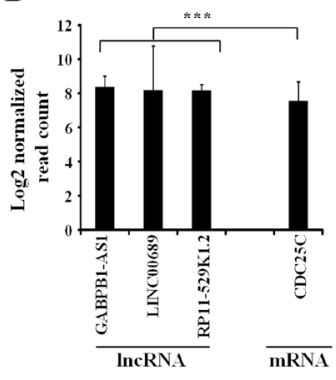

D

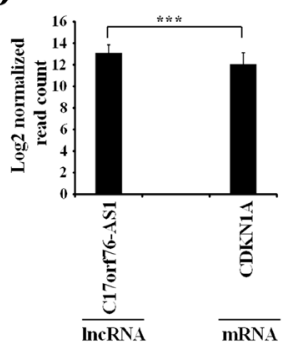

C

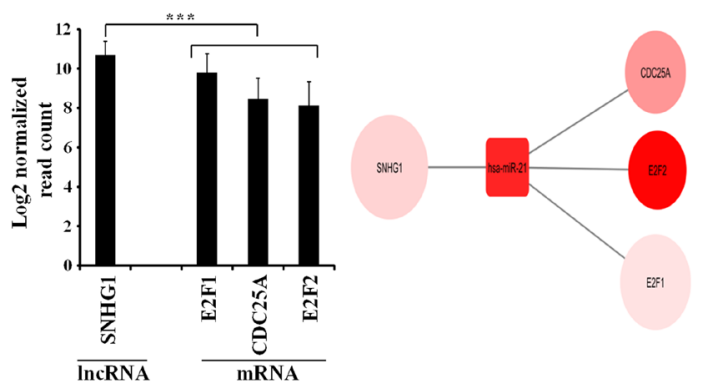

$\mathbf{E}$

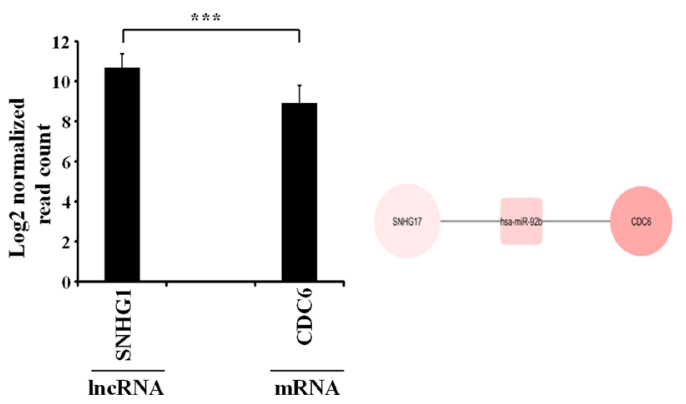

Figure 4: Competing endogenous RNAs (ceRNA) network in GBM. (A) Significantly enriched pathways in REACTOME, BIOCARTA and KEGG database for ceRNA network genes. (B, C, D and E) ceRNA network of four distinct networks related to Cell Cycle pathway in GBM. Bar graph shows the average $\log _{2}$ abundance of cell cycle network molecules in GBM samples with standard deviation. Square nodes represent miRNAs and circle nodes represent lncRNAs and mRNAs. The size of the node (lncRNA and mRNA) is proportional to average abundance of the molecule in GBM samples. Intensity of colour represents the $\log _{2}$ fold change between GBM samples and control brain samples. 
G-CIMP, IDH1 mutation, MGMT promoter methylation (Figure 7B). The distribution of lncRNA risk score and a comparison of risk score with patient survival are shown (Figure 7C and 7D). Further, lncRNA risk score stratified GBMs into low- and high-risk groups with significant difference in patient survival (median survival: 19.93 vs 11.17 months; Figure 7E). The expression pattern of five lncRNA between the low- and high-risk groups is shown (Figure 7F). SOX21-AS1 appears to act like a protective lncRNA as it expression was found to be higher in low risk group. In contrast, the other four lncRNAs appear to be risky lncRNAs as their expression is more in high-risk group. While the G-CIMP+ and IDH1 mutation samples entirely belong to low-risk group, the MGMT promoter methylation status and gene expression sub types appear to be equally distributed between low- and high-risk groups.

\section{DISCUSSION}

Aberrant transcription, a hallmark of cancer, is responsible for the dysregulation of transcripts in the cell. The differential expression of lncRNAs in cancer is implicative of their role in the malignancy [14-16]. However, there also exists the possibility that their altered expression could be an outcome of the disease, with no consequential role in its malignancy. Hence using lncRNA expression profiling, we have sought to identify lncRNAs whose aberrant expressions might translate to functions, thereby narrowing down on those with physiological relevance. Based on the known functions of lncRNAs, we used attributes such as their sequence and expression features to computationally narrow down on the prospective roles of the differentially expressed lncRNAs.

Microarray profiling studies of IncRNA genes in glioma has led to the discovery of several transcripts that have been used for subtype classification [29-31] and for the development of molecular signatures [32]. However, most of these studies have been conducted with the utilization of mined data from existing arrays that have coincidental representation of lncRNA probes. This approach faces the limitation of receiving an underrepresentation of the dysregulated lncRNAs in the disease. Our study that has employed the analysis of over 30,000 lncRNAs, therefore offers a highly comprehensive estimation of the long noncoding RNAs whose expressions are disrupted in GBM. Further, it is noteworthy that we have included a larger cohort for the study, comprising of 19 GBM patient samples, as compared to other studies $[33,34]$. We identified a total of $7,790 \mathrm{lncRNA}$ transcripts of which 2,774 transcripts were upregulated and 5,016

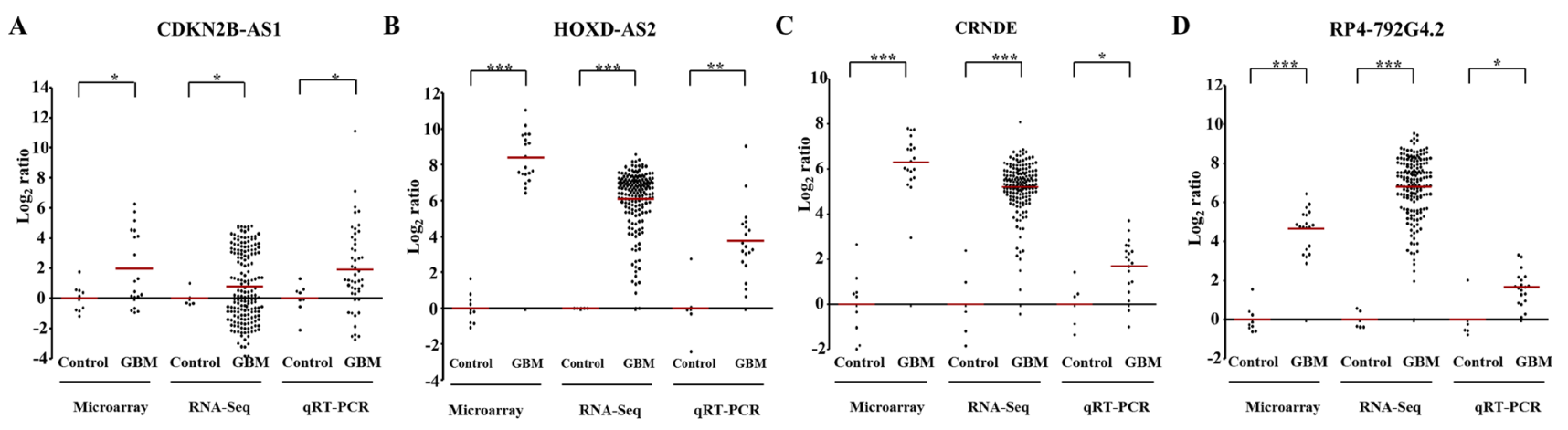

$\mathbf{E}$

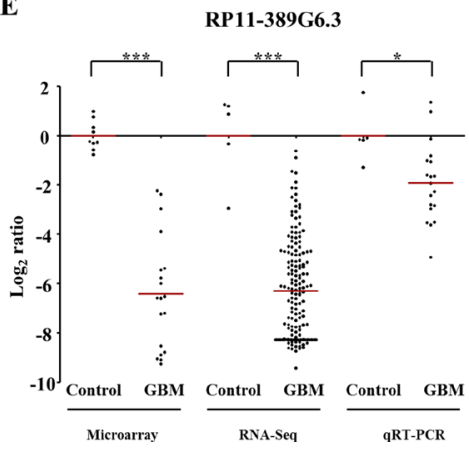

F

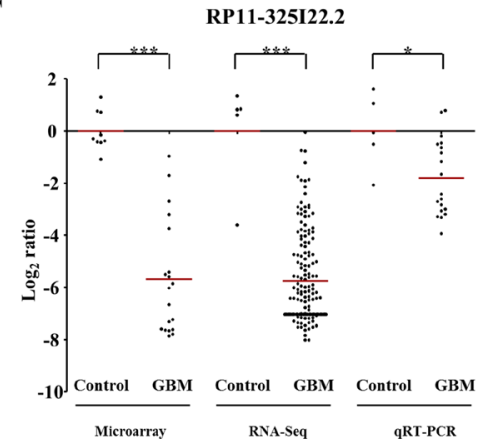

G

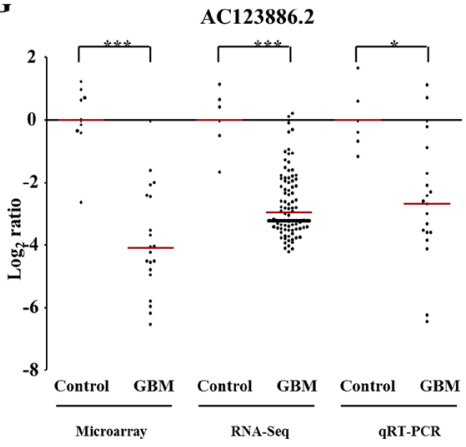

Figure 5: Validation of differentially regulated IncRNAs by RNA sequencing and real time qPCR. The expression of lncRNAs in TCGA cohort (RNA sequencing) and our cohort (RT-qPCR) as compared to microarray data (our cohort), has been depicted as scatter plots for CDKN2B-AS1 (A), HOXD-AS2 (B), CRNDE (C), RP4-792G4.2 (D), RP11-389G6.3 (E), RP11-325I22.2 (F) and RP11-325I22.2 (G). Each dot represents the data derived from one sample. For each sample, fold change in expression is calculated over its average expression in control brain tissue. Our cohort was a subset of the samples subjected to microarray analysis. The $p$ values have been represented by ${ }^{* * * *}$ and ${ }^{* * *}$ which denotes values of $p<0.05, p<0.01$ and $p<0.001$ respectively. 
were downregulated. The presence of several lncRNAs like CRNDE, HOTAIRM1 and H19 among the upregulated transcripts, were in concordance with earlier reports on their oncogenic role in glioma $[6,30,33,35]$. Similarly MEG3, a lncRNA with tumour suppressor properties [17], was downregulated in our microarray results. The dysregulated lncRNAs could segregate the control brain samples from the diseased ones, thus indicating their potential prominence in the disease. The vast numbers of dysregulated lncRNA transcripts which ran into thousands, prompted us to subject them to further analysis that would reveal their other significant functional attributes.

The reported mechanisms of IncRNA function majorly involve their regulation of the expression of genes, that may be either proximal to the lncRNA gene or may even be on a different chromosome. However, proximity to a gene need not render the lncRNA a regulator. In addition, regulation of distant genes has been reported to be dependent on their structure and not sequence, as in the case of the lncRNA MEG3 [36]. Therefore, neither the proximity to a gene nor its sequence similarity can implicate the targets of its regulation. We hence used a 'guilt by association' strategy [37] and carried out lncRNA-mRNA coexpression analysis on a sample to sample basis. The genomic distance of $500 \mathrm{~kb}$ we chose for the estimation of the regulations in cis, was on the basis of several reports on enhancer effects over a broader genomic region as in the case of HOTTIP,
A

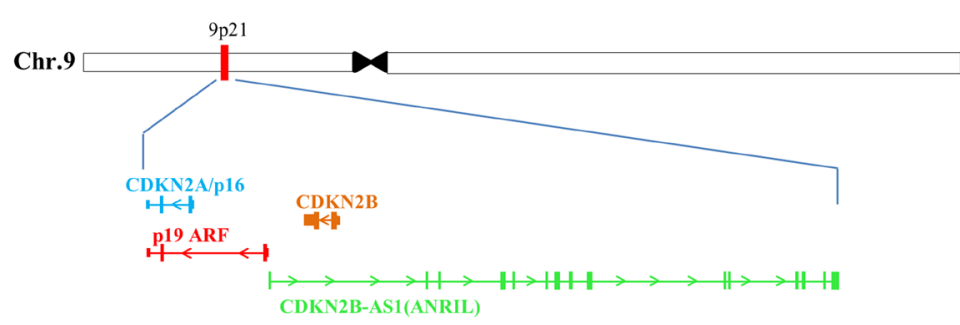

C

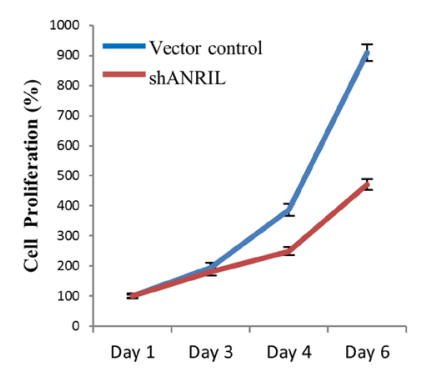

F

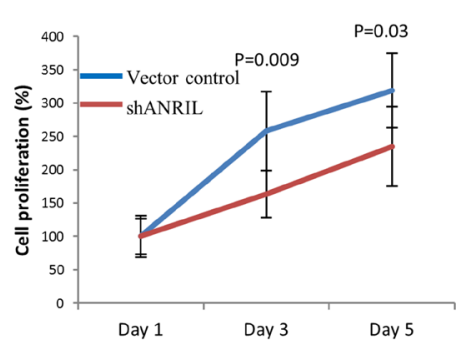

D

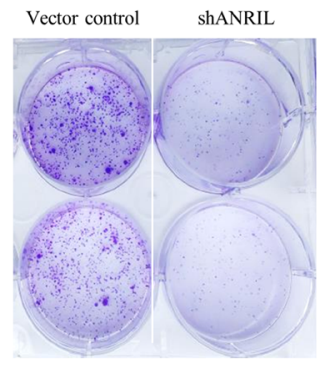

G

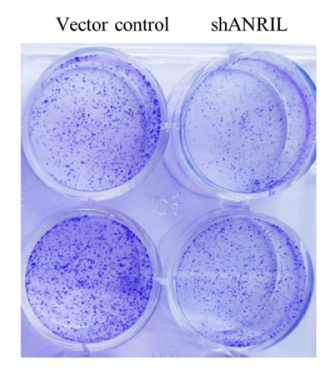

B

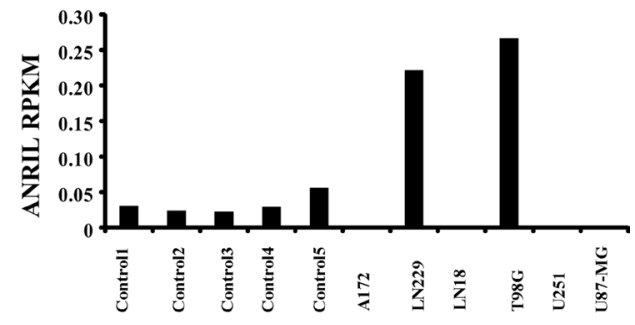

$\mathbf{E}$
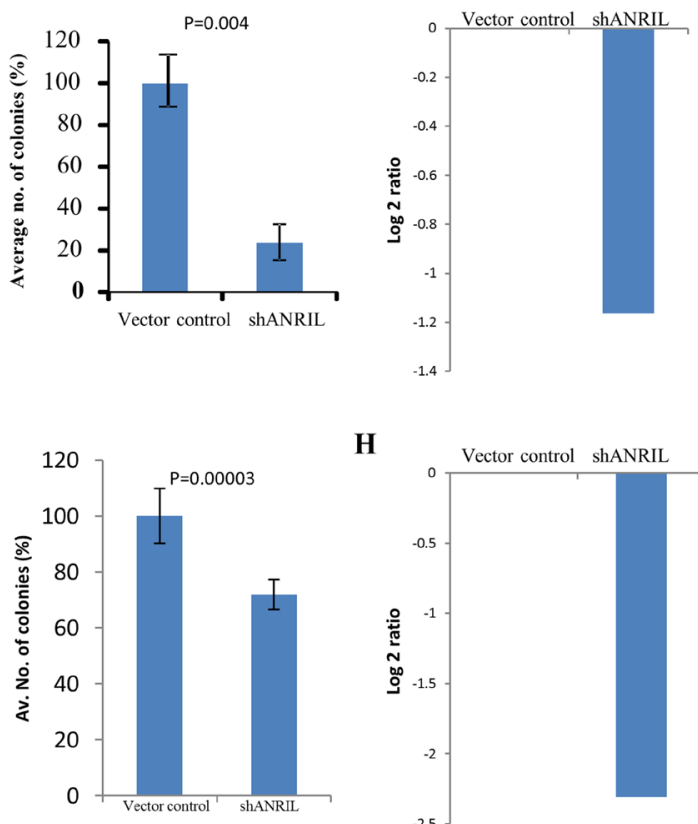

H

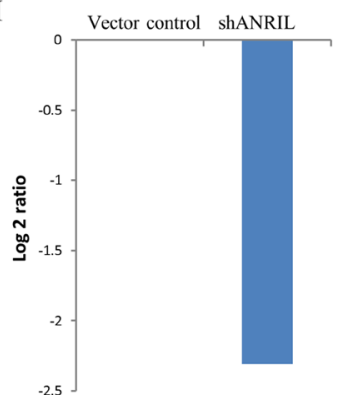

Figure 6: ANRIL is upregulated in GBM and its downregulation results in the inhibition of proliferation. (A) A snapshot depicting the genomic location of ANRIL gene in the intronic anti-sense region of $C D K N 2 B$ gene. (B) Column plot indicating the expression of ANRIL ( $\log _{2}$ ratio) across different glioma cell lines compared to control brain tissue. (C) LN229 cells were transfected with vector control or shANRIL, plated for the proliferation assay and the relative proliferation was quantified by MTT assay. (D) LN229 cells were transfected with vector control or shANRIL. The cells were then plated in duplicates in a 6-well plate. After 2 weeks of plating, the colonies were stained with crystal violet, photographed (left) and counted (right). $\%$ colony density \pm sd is plotted. (E) $\log _{2}$ transformed levels of ANRIL transcript upon $48 \mathrm{hrs}$ of shRNA knockdown in LN229 cell line. (F) T98G cells were transfected with vector control or shANRIL, plated for the proliferation assay and the relative proliferation was quantified by trypan blue assay. (G) T98G cells were transfected with vector control or shANRIL. The cells were then plated in duplicates in a 6-well plate. After 2 weeks of plating, the colonies were stained with crystal violet, photographed (left) and counted (right). \% colony density \pm sd is plotted. (H) $\log _{2}$ transformed levels of ANRIL transcript upon $48 \mathrm{hrs}$ of shRNA knockdown in T98G cell line. 
ecCEBPA, Mistral and Air [9, 21, 38]. Several hundred of IncRNA-mRNA pairs, with correlation between them both positively and negatively, were obtained in cis and trans as well. It is worth noting several mRNAs coding for proteins relevant to transformation including tumor suppressors, oncogenes, kinases, pro and anti apoptotic proteins. To the best of our knowledge, our analysis on the co-regulation of lncRNAs is the first comprehensive analysis of its kind till date. These correlations would give insight into additional layers of regulation of the lncRNAmRNA association network.

The regulation exerted by miRNAs in the cell is complex and profound due to the propensity of a single miRNA to regulate several transcripts. Their influence therefore extends to the various biological processes in the cell and consequently to tumour progression. There is significant crosstalk between miRNAs, IncRNAs and
mRNAs in the cell, which when depicted as networks, is highly complex. The roles of lncRNAs in this network majorly include their ability to serve either as a source of miRNAs or as a sequester of the miRNAs. H19 has been reported to be the source of miR-675, which targets Insulin growth factor receptor [39]. By mapping the coordinates of the miRNAs to those of dysregulated IncRNAs, we could identify the lncRNAs that harboured miRNAs. From the several lncRNAs obtained as potential miRNA hosts, we could demonstrate that differential regulation of lncRNA as the reason for differential regulation of miRNA. The upregulated MIR17HG gives rise to the miR 17/92 cluster, which is well studied in cancer and in glioblastoma [40] In the next exercise, we obtained several lncRNAs that showed complementarity with the mature sequence of miRNAs. Their opposite regulation is indicative of their potential role as sponges for the miRNAs [11, 41]. We
A

\begin{tabular}{|l|c|c|c|c|c|}
\hline \multicolumn{1}{|c|}{ IncRNA } & $\begin{array}{c}\text { No. of } \\
\text { patients }\end{array}$ & $\begin{array}{c}\text { Hazard } \\
\text { Ratio }\end{array}$ & $\begin{array}{c}\text { B } \\
\text { (coefficient) }\end{array}$ & p value & Prognosis \\
\hline SOX21-AS1 & 152 & 0.713 & -0.339 & 0.001 & Good \\
\hline RP6-99M1.2 & 152 & 1.309 & 0.270 & 0.001 & Poor \\
\hline CTD-2127H9.1 & 152 & 1.304 & 0.265 & 0.001 & Poor \\
\hline RP11-375B1.3 & 152 & 1.291 & 0.255 & 0.001 & Poor \\
\hline RP3-449M8.9 & 152 & 1.384 & 0.325 & 0.002 & Poor \\
\hline
\end{tabular}

B

\begin{tabular}{|c|c|c|c|c|}
\hline Factor & $\begin{array}{c}\text { No. of } \\
\text { patients }\end{array}$ & $\begin{array}{c}\text { Hazard } \\
\text { Ratio }\end{array}$ & B & $\mathrm{p}$ value \\
\hline \multicolumn{5}{|c|}{ I. Univariate analysis TCGA dataset } \\
\hline Age & 152 & 1.034 & 0.034 & $<0.001$ \\
\hline G-CIMP & 151 & 0.204 & -1.589 & 0.027 \\
\hline IDH status & 149 & 0.240 & -1.428 & 0.046 \\
\hline MGMT status & 121 & 0.640 & -0.446 & 0.068 \\
\hline LncRNA Risk score & 152 & 2.046 & 0.716 & $<0.001$ \\
\hline \multicolumn{5}{|c|}{ II. Multivariate analysis with TCGA dataset } \\
\hline Age & \multirow[t]{2}{*}{152} & 1.030 & 0.030 & 0.002 \\
\hline LncRNA Risk score & & 2.072 & 0.729 & $<0.001$ \\
\hline G-CIMP & \multirow[t]{2}{*}{151} & 0.505 & -0.683 & 0.358 \\
\hline LncRNA Risk score & & 1.906 & 0.645 & $<0.001$ \\
\hline IDH status & \multirow[t]{2}{*}{149} & 0.596 & -0.518 & 0.484 \\
\hline LncRNA Risk score & & 1.987 & 0.687 & $<0.001$ \\
\hline MGMT status & \multirow[t]{2}{*}{121} & 1.077 & 0.074 & 0.782 \\
\hline LncRNA Risk score & & 2.261 & 0.816 & $<0.001$ \\
\hline \multicolumn{5}{|c|}{ III. Multivariate analysis of all the markers in TCGA dataset } \\
\hline Age & \multirow[t]{5}{*}{118} & 1.045 & 0.044 & $<0.001$ \\
\hline G-CIMP & & 0.000 & -8.837 & 0.962 \\
\hline IDH status & & & 9.012 & 0.961 \\
\hline MGMT status & & 1.132 & 0.124 & 0.648 \\
\hline LncRNA Risk score & & 2.214 & 0.795 & $<0.001$ \\
\hline
\end{tabular}

C

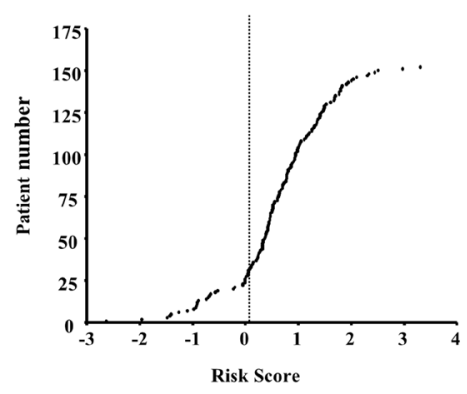

D

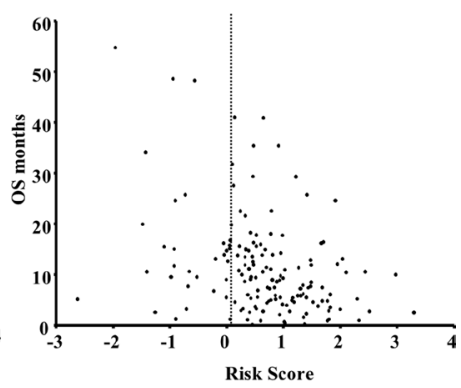

$\mathbf{E}$

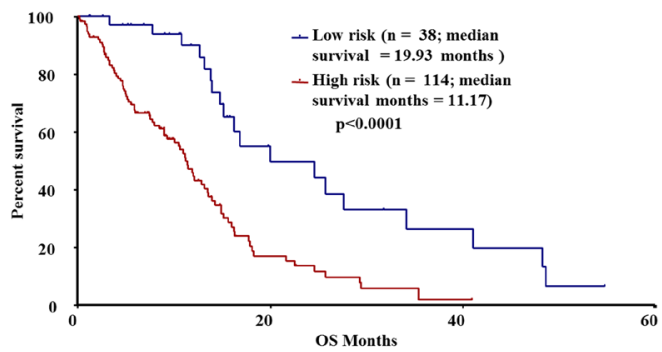

F

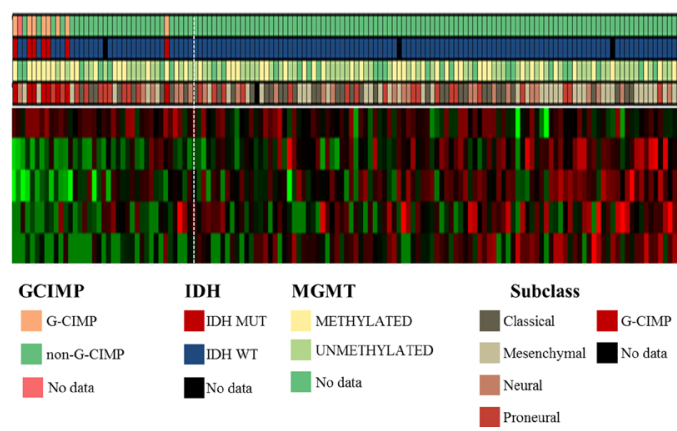

Figure 7: Prognostic role of IncRNA signature in GBM. (A) Univarate Cox proportional hazard regression analysis of five lncRNAs using TCGA RNA-Seq. data. (B) Univariate and multivariate Cox proportional hazard regression analysis of lncRNA signature risk score with age, G-CIMP methylation status, IDH mutation and MGMT promoter methylation status. (C) lncRNA risk score distribution of the GBM patients from TCGA RNA-Seq cohort. (D) Patient survival status along with risk score from TCGA RNA sequencing cohort. (E) Kaplan-Meier survival estimates overall survival of GBM patients according to the lncRNA expression signature. (F) Heat map of five lncRNAs expression profiles of GBM patients; rows represent lncRNAs, and columns represent patients. The dotted white line represents the lncRNA signature cut-off dividing patients into low-risk and high-risk groups. 
were able to carry out extensive analysis to identify physiologically relevant lncRNA-miRNA-mRNA sponges wherein the lncRNAs are more likely to work as sponges in titrating miRNAs such that target mRNAs are spared from miRNA effects. It is interesting to note that a set of genes related to cell cycle and antigen presentation in particular are regulated by lncRNA sponges.

Several lncRNAs reportedly having crucial roles in cancer, including HOTAIR, MALAT1 and H19, have been reported to be linked to survival $[5,42,43]$. This encouraged us to look into the propensity of the dysregulated lncRNAs to function as prognostic tools in GBM. This analysis revealed several lncRNAs could act as poor or good prognostic markers. Further, we demonstrate that a lncRNA rick-score based on top five lncRNAs is an independent predictor of survival in GBM. We were able to stratify GBMs into low and high-risk groups with significant difference in the survival.

The IncRNA ANRIL has been shown to be upregulated and repress INK4b-ARF-INK4a locus epigenetically by recruiting CBX7 in prostate cancer [27]. While we found ANRIL is upregulated in GBM overall, we found that it is co-deleted in GBMs with homozygous deletion of INK4b-ARF-INK4a locus. In GBMs with no copy number aberration in INK4b-ARF-INK4a locus, we found upregulation of all genes that are present in the locus -ANRIL, CDKN2A and CDKN2B thus signifying the absence of ANRIL-mediated repression of INK4b-ARFINK4a locus in GBM. On possible reason for the absence of repression of INK4b-ARF-INK4a locus by ANRIL is the downregulation of the co-repressor molecule CBX7 in GBMs. Thus our results demonstrate that ANRIL is upregulated in GBM and is an oncogeneic lnRNA in GBM. Further, unlike what is shown in prostate cancer [27], ANRIL is not likely to be the repressor of INK4b/ARF/ INK4a locus in GBM.

This unique in-silico functional characterization of lncRNAs provides us the very first comprehensive glimpse of lncRNA regulatory effects in GBM. This study can form the basis and provide direction for several future endeavours that aim to dissect the layer of regulation exerted by lncRNAs in GBM. IncRNAs have been shown to function as therapeutic targets in many diseases. This is of particular importance in cancers like GBM wherein clinicians face difficulty in devising effective treatment strategies. Exploring the functional relevance of lncRNAs in GBM will help give better insights into the deregulated pathways of this disease.

\section{MATERIALS AND METHODS}

\section{Tumor samples and clinical details}

Tumor samples were collected from patients who were operated at National Institute of Mental Health and Neurosciences (NIMHANS) and Sri Satya Sai Institute of
Higher Medical Sciences (SSSIHMS), Bangalore, India. A portion of the anterior temporal cortex resected during surgery for drug resistant epilepsy patients served as control brain sample. The study was scrutinized and approved by the ethics committee of the two clinical centres, NIMHANS and SSSIHMS, and patient consent was taken before initiation of the study as per Institute Ethical Committee guidelines and approval. We used a total of 19 GBM patient samples and 9 control brain tissue samples for this study. The histology was confirmed as GBM by the neuropathologist as per WHO 2007 classification scheme.

\section{Cell lines and plasmid}

The glioma cell lines (A172, LN229, LN18, T98G, U251 and U87-MG) used in this study were obtained from Sigma Aldrich, Saint Louis, Missouri, USA. These cells were grown in Dulbeccos modified Eagle's medium (DMEM) supplemented with 10\% FBS. The cells were maintained at $37^{\circ} \mathrm{C}$ and $5 \% \mathrm{CO}_{2}$ Retro-virus based shRNA construct for ANRIL (pSR_ANRIL) was a kind gift from Dr Yojiro Kotake, Kindai University, Japan [3]. The control retroviral vector (pSR_SCR) used was obtained from Addgene, USA.

\section{Tissue RNA isolation}

Total RNA was extracted from Glioblastoma (GBM) and control brain tissue samples. Briefly, the tissue was subjected to homogenisation in the presence of trizol reagent. Chloroform was added and the tubes were vortexed vigorously followed by centrifugation at 14,000 $\mathrm{rpm}$ for $30 \mathrm{~min}$. Isopropanol was used to precipitate the RNA from the aqueous phase. The pellet was washed with $70 \%$ ethanol and dissolved in triple autoclaved distilled water. RNA quantity and integrity was determined by nanodrop and by denaturing agarose gel electrophoresis.

\section{Arraystar microarray sample preparation and microarray}

Arraystar Human lncRNA Microarray V3.0 was used for the global profiling of human lncRNAs and proteincoding transcripts. About 30,586 lncRNAs and 26,109 coding transcripts can be detected by this IncRNA microarray.

\section{RNA labelling and array hybridization}

Sample labelling and array hybridization were performed according to the Agilent One-Color MicroarrayBased Gene Expression Analysis protocol (Agilent Technology) with minor modifications. Briefly, mRNA was purified from total RNA after removal of rRNA (mRNAONLYTM Eukaryotic mRNA Isolation Kit, Epicentre). Then, each sample was amplified and transcribed into fluorescent cRNA along the entire length of the transcripts without 3' bias utilizing a random priming method (Arraystar Flash 
RNA Labeling Kit, Arraystar). The labelled cRNAs were purified by RNeasy Mini Kit (Qiagen). The concentration and specific activity of the labelled cRNAs (pmol Cy3/ $\mu \mathrm{g}$ cRNA) were measured by NanoDrop ND-1000. $1 \mu \mathrm{g}$ of each labelled cRNA was fragmented by adding $5 \mu \mathrm{l} 10 \times$ Blocking Agent and $1 \mu$ of $25 \times$ Fragmentation Buffer, then heated the mixture at $60^{\circ} \mathrm{C}$ for $30 \mathrm{~min}$, finally $25 \mu \mathrm{L} 2 \times$ GE Hybridization buffer was added to dilute the labelled cRNA. $50 \mu \mathrm{l}$ of hybridization solution was dispensed into the gasket slide and assembled to the lncRNA expression microarray slide. The slides were incubated for 17 hours at $65^{\circ} \mathrm{C}$ in an Agilent Hybridization Oven. The hybridized arrays were washed, fixed and scanned with using the Agilent DNA Microarray Scanner (part number G2505C).

\section{Microarray data analysis}

Agilent Feature Extraction software (version 11.0.1.1) was used to analyze acquired array images. Quantile normalization and subsequent data processing were performed with using the GeneSpring GX v12.1 software package (Agilent Technologies). After quantile normalization of the raw data, IncRNAs and mRNAs that at least 7 out of 28 samples have flags in Present or Marginal ("All Targets Value") were chosen for further data analysis. Differentially expressed lncRNAs were identified by calculating student $t$ test between GBM samples and control brain samples (Fold Change $\geq 2.0, p$ value $<0.05$ ).

\section{TCGA RNA sequencing analysis}

We obtained raw RNA sequencing data for GBM samples from TCGA. The whole RNA sequencing data was aligned using PRADA tool [44]. Duplicate removal was carried out using Picard 1.73 [45]. IncRNAs were annotated as per Gencode Version 19 annotation file [28]. The RNA-seq reads were counted over gene exons using HtSeq [46]. We used the DESeq2 size factor correction to account for differences in sequencing depth between the samples [47].

\section{IncRNA survival analysis}

The expression level of each lncRNA was used to check there correlation with survival. The 152 GBM samples (TCGA RNA-seq cohort) for which survival information were available were used for univariate Cox regression analysis using survival package of $\mathrm{R}$ (version 3.2.3). SPSS version 19.0 was used for multivariate analysis. Kaplan Meier survival analysis was performed using GraphPad Prism 5.0 version for Windows (GraphPad Software, San Diego, California USA, www.graphpad. com). The risk score for the signature was calculated using the following formula:

Risk score of a sample $=\sum$ (cox regression coefficient of a particular lncRNA $X \log _{2}$ value of expression of lncRNA).

\section{Integrative analysis of IncRNAs in GBM}

\section{LncRNAs having enhancer like functions that regulate mRNAs in cis and trans}

Sample wise Spearman correlation was performed between all the differentially expressed IncRNAs $(n=4,289)$ and differentially expressed mRNAs ( $n=5,076)$ for 19 GBM samples (our cohort) using $\mathrm{R}$ software (version 3.1.0). This analysis resulted in 21,770,964 lncRNA-mRNA pairs. IncRNA-mRNA pairs were divided into two groups (a) cis pairs (mRNAs that lie $500 \mathrm{~kb}$ within upstream and downstream of the lncRNA coordinates) and (b) trans (mRNAs that lie $>500 \mathrm{~kb}$ upstream and downstream of the lncRNA coordinates). Out of 10,968 lncRNA-mRNA cis pairs, 2,674 lncRNA-mRNA cis pairs were significantly correlated ( $p$ value $<0.05$ ). Since the lncRNA-mRNA trans pairs were large in number ( $n=21,759,996)$, we used stringent cut off ( $p$ value $<0.05$ and $|r|>0.9)$. We found 4,679,124 lncRNA-mRNA trans pairs were significantly $(p$ value $<0.05$ ) correlated. Out of these pairs 24,631 lncRNA-mRNA trans pairs were having absolute Spearman correlation coefficient greater than 0.9 (Supplementary Figure 3).

\section{LncRNAs regulating IncRNAs}

Sample wise Spearman correlation was performed for all the differentially expressed $\operatorname{lncRNAs}(n=4,289)$ for 19 GBM samples (our cohort) using R software (version 3.1.0). From this analysis we found a total of $18,395,521$ lncRNA-lncRNA pairs. Correlation analysis of an IncRNA with itself would yield $R$ value of 1 . Hence, to obtain the true IncRNA-lncRNA pairs, we subtracted the above correlation pairs $(n=4,289)$ to give $18,391,232$ lncRNAlncRNA pairs. IncRNA-lncRNA pairs were divided into two groups (a) cis pairs (lncRNAs that lie $500 \mathrm{~kb}$ within upstream and downstream of the other lncRNA coordinates) and (b) trans (lncRNAs that lie $>500 \mathrm{~kb}$ upstream and downstream of the other lncRNA coordinates). A total of 3,386 IncRNA-IncRNA cis pairs were obtained that were significantly correlating ( $p$ value $<0.05$ ). However, this included correlation between lncRNA pairs twice (e.g.: A vs $\mathrm{B}$ and $\mathrm{B}$ vs A). Hence, to obtain the unique pairs, only one of the correlation values between IncRNA pairs were considered to obtain 1,693 lncRNA-lncRNA cis pairs. Since lncRNA-lncRNA trans pairs were large in number $(n=$ $18,378,940$ ), we used stringent cut off ( $p$ value $<0.05$ and $|r|>0.9$ ). We found 3,216,060 lncRNA-lncRNA trans pairs were significantly correlated ( $p$ value $<0.05$ ). Out of these pairs 8,547 lncRNA-lncRNA trans pairs were unique and having absolute Spearman correlation coefficient greater than 0.9 (Supplementary Figure 4).

\section{LncRNAs as miRNA hosts}

Further, we wanted to identify long non-coding RNAs that might give rise to small non-coding RNAs, 
that is, micro RNAs. For this; genomic coordinates of deregulated lncRNAs from Arraystar microarray analysis were mapped to the genomic coordinates of the list of 2794 miRNAs downloaded from miRBase (http://www.mirbase. org/ftp.shtml; version 21) [48, 49]. This was performed using "Intersect" function of BEDtools. Several miRNAs and miRNA families were identified; to which differentially expressed lncRNAs in GBM were playing host to- in the form of parent gene. To this we added the miRNA expression data in GBM from TCGA miRNA microarray as well as miRNA expression data from the microarray performed previously for a separate cohort in lab. This enabled us to identify significantly regulated miRNAs in GBM whose parent gene is a deregulated lncRNA in GBM. The regulation of such miRNAs can be thought of being regulated by the parent gene; although further investigation into whether the small non-coding RNA shares its promoter with that of the lncRNA is required.

\section{LncRNAs as miRNA decoys}

The competing endogenous RNAs (ceRNAs) network was constructed to identify lncRNA-miRNA-mRNA sponge modules by the following step: 1) differentially expressed lncRNAs between GBM and control brain samples from TCGA RNA-seq were taken ( $n=1,559$; fold change $\geq 1.5 \&$ FDR $<0.05)$. 2) miRcode [50] was used to predict miRNAs that target the differentially expressed lncRNAs, 3) miRNAs obtained from the above analysis was used to obtain their experimentally validated target mRNAs extracted from miRWalk [51].

Out of the differentially regulated lncRNAs ( $n=1,559)$, a total of 1,092 lncRNAs were present in miRcode database. From miRcode database, we obtained 26,620 lncRNA-miRNA pairs having expression data in TCGA GBM samples of which 2,531 lncRNA-miRNA pairs ( $\operatorname{lncRNA} n=695$; miRNA $n=133$ ) showed negative correlation. Next, miRNAs obtained from the above analysis was used to detect their experimentally validated mRNA targets obtained from miRWalk. From this analysis, we obtained 47,887 miRNA-mRNA pairs having expression data in TCGA GBM samples from which 5,336 miRNA-mRNA pairs having negative correlation (miRNAs $n=125$; mRNA $n=3060$ ) were taken for consideration. In the next step, we merged the lncRNAmiRNA pairs $(n=2,531)$ with miRNA-mRNA pairs $(n=5,336)$ to create lncRNA-miRNA-mRNA sponge modules $(n=150,684)$. In the next step, from these sponge modules, we selected only those modules that showed a positive correlation between mRNA-lncRNA and all three (lncRNAs, miRNA and mRNA) showed upregulation in GBM compared to control samples $(n=9,448)$. In the last step, those modules wherein the abundance of IncRNA transcript levels is significantly higher than that of mRNA which resulted in a true lncRNA-miRNA-mRNA sponge module $(n=408)$, wherein the lncRNA is highly likely to work as a sponge such that the target mRNA will be spared for the miRNA. The unique set of mRNAs $(n=$ 134) from this finally selected lncRNA-miRNA-mRNA sponge modules were used for DAVID pathway analysis to find out important pathways that are regulated by these sponge modules.

\section{LncRNA validation by real-time PCR}

The RNA was reverse transcribed using HighCapacity cDNA Reverse transcription kit (Thermo Fisher Scientific). Dynamo SYBR qRT-PCR kit (Finnzymes) was used for real- time PCR assays. The mRNA levels of RPL35 and ATP5G were used as endogenous controls. Specific primer pairs for the lncRNAs were ordered from Sigma Aldrich and their sequences (5'-3') are as follows - HOXD-AS2: CAAAGGAACTGCTCTGGTGA, CCAAGCTTCTTGTGTCCTCTG; CRNDE: TCATGA TTAGCAGGCAGACG, ACAAACGGTCACCACTAC CC; RP11- 389G6.3: CAATATGCAGGATGGGAAGG, CCAGAGTCCTTGGAAACCAC; RP11-325I22.2: AAT ACGGGTTGAGCATCAGG, AATCGCCATCCTTTCAC AAC; ANRIL: TTTCCTACGAAGCTGGGTGA, GTAA AACGCAACAAGATAGAGAAGC.

\section{ANRIL silencing}

LN229 and T98G cells were seeded at the density of 0.8 million in $60 \mathrm{~mm}$ petri dish. After 16 hours of initial seeding, cells were transfected with $8 \mu \mathrm{g}$ of pSR_ANRIL and control plasmids using opti-MEM and lipofectamine reagents. After 6 hours of transfection, opti-MEM was replaced by complete DMEM (DMEM+10\% FBS). Post 48 hours of transfection, cells were harvested, counted and seeded for proliferation and colony forming ability. The knockdown of ANRIL in the transfected cells over the vector control transfected cells was confirmed by qRT- PCR.

\section{Cell proliferation by MTT}

Cells either transfected with pSR_ANRIL or control plasmids were harvested and plated at density of 1500 cells per well in 96 well plate in triplicates. Each day a triplicate set for each condition were treated with $10 \mu \mathrm{l}$ (5 mg/ml in PBS) of MTT [3-(4,5-Dimethylthiazol-2-yl)2,5-Diphenyltetrazolium Bromide] to quantify the viable cell number. Three hours after addition of MTT, formazan crystal were dissolved in $200 \mu \mathrm{l}$ of DMSO, mixed well and absorbance was measured at $570 \mathrm{~nm}$ using Elisa plate reader. The statistical significance were calculted by student's $T$ test.

\section{Cell proliferation by trypan blue}

Cells either transfected with pSR_ANRIL or control plasmids were harvested and plated at density of 30000 cells per well in 12 well plate in duplicates. Each day, 
a duplicate set was harvested, washeshed with PBS, resuspended in $0.32 \%$ trypan blue and viable cells were counted using haemocytometer. statistical significance were calculted by student's $T$ test.

\section{Colony formation assay}

Cells either transfected with pSR_ANRIL or control plasmids were harvested and plated at density of 2000 cells per well in 6 well plate in triplicates. Every third day, the culture media were replaced. After 12 days of culturing, the colonies were fixed with $100 \%$ chilled methanol for $30 \mathrm{~min}$ and then stained with $0.05 \%$ crystal violet for $30 \mathrm{~min}$ and photographed.

\section{Abbreviations}

lncRNA: long non-coding RNA; ceRNA: competing endogenous RNA; ANRIL: antisense non-coding RNA in the INK4 locus; GBM: Glioblastoma; CNV: Copy Number Variation; TCGA: The Cancer Genome Atlas; miRNA: Micro RNA; HR: Hazard Ratio; G-CIMP: Glioma-CpG island methylator phenotype

\section{ACKNOWLEDGMENTS}

The results published here are in whole or part based upon data generated by The Cancer Genome Atlas pilot project established by the NCI and NHGRI. Information about TCGA and the investigators and institutions that constitute the TCGA research network can be found at http://cancergenome.nih.gov/. KS acknowledges DBT, DST and CEFIPRA, Government of India for Research grant. Infrastructure support by funding from DST-FIST, DBT grant-in-aid and UGC (Centre for Advanced Studies in Molecular Microbiology) to MCB is acknowledged. ST acknowledges DBT, Government of India for the Post-doctoral fellowship. KS is a J. C. Bose Fellow of the Department of Science and Technology.

\section{CONFLICTS OF INTEREST}

Authors declare no conflicts of interest.

\section{REFERENCES}

1. Mercer TR, Mattick JS. Structure and function of long noncoding RNAs in epigenetic regulation. Nat Struct Mol Biol. 2013; 20:300-7. https://doi.org/10.1038/nsmb.2480.

2. Thrash-Bingham CA, Tartof KD. aHIF: a natural antisense transcript overexpressed in human renal cancer and during hypoxia. J Natl Cancer Inst. 1999; 91:143-51.

3. Kotake Y, Nakagawa T, Kitagawa K, Suzuki S, Liu N, Kitagawa M, Xiong Y. Long non-coding RNA ANRIL is required for the PRC2 recruitment to and silencing of
p15(INK4B) tumor suppressor gene. Oncogene. 2011; 30:1956-62. https://doi.org/10.1038/onc.2010.568.

4. Kino T, Hurt DE, Ichijo T, Nader N, Chrousos GP. Noncoding RNA gas5 is a growth arrest- and starvation-associated repressor of the glucocorticoid receptor. Sci Signal. 2010; 3:ra8. https://doi.org/10.1126/scisignal.2000568.

5. Ji P, Diederichs S, Wang W, Böing S, Metzger R, Schneider PM, Tidow N, Brandt B, Buerger H, Bulk E, Thomas M, Berdel WE, Serve H, Müller-Tidow C. MALAT-1, a novel noncoding RNA, and thymosin beta4 predict metastasis and survival in early-stage non-small cell lung cancer. Oncogene. 2003; 22:8031-41. https://doi. org/10.1038/sj.onc.1206928.

6. Shi Y, Wang Y, Luan W, Wang P, Tao T, Zhang J, Qian J, Liu N, You Y. Long non-coding RNA H19 promotes glioma cell invasion by deriving miR-675. PLoS One. 2014; 9:e86295. https://doi.org/10.1371/journal.pone.0086295.

7. Tomita S, Abdalla MO, Fujiwara S, Matsumori H, Maehara K, Ohkawa Y, Iwase H, Saitoh N, Nakao M. A cluster of noncoding RNAs activates the ESR1 locus during breast cancer adaptation. Nat Commun. 2015; 6:6966. https://doi. org/10.1038/ncomms7966.

8. Lam MT, Li W, Rosenfeld MG, Glass CK. Enhancer RNAs and regulated transcriptional programs. Trends Biochem Sci. 2014; 39:170-82. https://doi.org/10.1016/j. tibs.2014.02.007.

9. Wang KC, Yang YW, Liu B, Sanyal A, CorcesZimmerman R, Chen Y, Lajoie BR, Protacio A, Flynn RA, Gupta RA, Wysocka J, Lei M, Dekker J, et al. A long noncoding RNA maintains active chromatin to coordinate homeotic gene expression. Nature. 2011; 472:120-4. https:// doi.org/10.1038/nature09819.

10. Croce CM. Causes and consequences of microRNA dysregulation in cancer. Nat Rev Genet. 2009; 10:704-14. https://doi.org/10.1038/nrg2634.

11. Cesana M, Cacchiarelli D, Legnini I, Santini T, Sthandier O, Chinappi M, Tramontano A, Bozzoni I. A long noncoding RNA controls muscle differentiation by functioning as a competing endogenous RNA. Cell. 2011; 147:358-69. https://doi.org/10.1016/j.cell.2011.09.028.

12. Johnsson P, Ackley A, Vidarsdottir L, Lui WO, Corcoran M, Grander D, Morris KV. A pseudogene long-noncoding-RNA network regulates PTEN transcription and translation in human cells. Nat Struct Mol Biol. 2013; 20:440-6. https:// doi.org/10.1038/nsmb.2516.

13. Cheng EC, Lin H. Repressing the repressor: a lincRNA as a MicroRNA sponge in embryonic stem cell selfrenewal. Dev Cell. 2013; 25:1-2. https://doi.org/10.1016/j. devcel.2013.03.020.

14. Du Z, Fei T, Verhaak RG, Su Z, Zhang Y, Brown M, Chen Y, Liu XS. Integrative genomic analyses reveal clinically relevant long noncoding RNAs in human cancer. Nat Struct Mol Biol. 2013; 20:908-13. https://doi.org/10.1038/ nsmb.2591. 
15. Iyer MK, Niknafs YS, Malik R, Singhal U, Sahu A, Hosono Y, Barrette TR, Prensner JR, Evans JR, Zhao S, Poliakov A, Cao X, Dhanasekaran SM, et al. The landscape of long noncoding RNAs in the human transcriptome. Nat Genet. 2015; 47:199-208. https://doi.org/10.1038/ng.3192.

16. Yan X, Hu Z, Feng Y, Hu X, Yuan J, Zhao SD, Zhang Y, Yang L, Shan W, He Q, Fan L, Kandalaft LE, Tanyi JL, et al. Comprehensive Genomic Characterization of Long Noncoding RNAs across Human Cancers. Cancer Cell. 2015; 28:529-40. https://doi.org/10.1016/j.ccell.2015.09.006.

17. Wang P, Ren Z, Sun P. Overexpression of the long non-coding RNA MEG3 impairs in vitro glioma cell proliferation. J Cell Biochem. 2012; 113:1868-74. https:// doi.org/10.1002/jcb.24055.

18. Ellis BC, Molloy PL, Graham LD. CRNDE: A Long Non-Coding RNA Involved in CanceR, Neurobiology, and DEvelopment. Front Genet. 2012; 3:270. https://doi. org/10.3389/fgene.2012.00270.

19. Reon BJ, Anaya J, Zhang Y, Mandell J, Purow B, Abounader R, Dutta A. Expression of IncRNAs in LowGrade Gliomas and Glioblastoma Multiforme: An In Silico Analysis. PLoS Med. 2016; 13:e1002192. https://doi. org/10.1371/journal.pmed.1002192.

20. Liao Q, Liu C, Yuan X, Kang S, Miao R, Xiao H, Zhao G, Luo H, Bu D, Zhao H, Skogerbo G, Wu Z, Zhao Y. Largescale prediction of long non-coding RNA functions in a coding-non-coding gene co-expression network. Nucleic Acids Res. 2011; 39:3864-78. https://doi.org/10.1093/nar/ gkq1348.

21. Nagano T, Mitchell JA, Sanz LA, Pauler FM, FergusonSmith AC, Feil R, Fraser P. The Air noncoding RNA epigenetically silences transcription by targeting G9a to chromatin. Science. 2008; 322:1717-20. https://doi. org/10.1126/science. 1163802 .

22. Li W, Notani D, Ma Q, Tanasa B, Nunez E, Chen AY, Merkurjev D, Zhang J, Ohgi K, Song X, Oh S, Kim HS, Glass CK, Rosenfeld MG. Functional roles of enhancer RNAs for oestrogen-dependent transcriptional activation. Nature. 2013; 498:516-20. https://doi.org/10.1038/nature12210.

23. Erdmann VA, Szymanski M, Hochberg A, Groot N, Barciszewski J. Non-coding, mRNA-like RNAs database Y2K. Nucleic Acids Res. 2000; 28:197-200.

24. Rodriguez A, Griffiths-Jones S, Ashurst JL, Bradley A. Identification of mammalian microRNA host genes and transcription units. Genome Res. 2004; 14:1902-10. https:// doi.org/10.1101/gr.2722704.

25. Sherr CJ. Divorcing ARF and p53: an unsettled case. Nat Rev Cancer. 2006; 6:663-73. https://doi.org/10.1038/nrc1954.

26. Brennan CW, Verhaak RG, McKenna A, Campos B, Noushmehr H, Salama SR, Zheng S, Chakravarty D, Sanborn JZ, Berman SH, Beroukhim R, Bernard B, Wu CJ, et al, and TCGA Research Network. The somatic genomic landscape of glioblastoma. Cell. 2013; 155:462-77. https:// doi.org/10.1016/j.cell.2013.09.034.
27. Yap KL, Li S, Munoz-Cabello AM, Raguz S, Zeng L, Mujtaba S, Gil J, Walsh MJ, Zhou MM. Molecular interplay of the noncoding RNA ANRIL and methylated histone $\mathrm{H} 3$ lysine 27 by polycomb CBX7 in transcriptional silencing of INK4a. Mol Cell. 2010; 38:662-74. https://doi. org/10.1016/j.molcel.2010.03.021.

28. http://www.gencodegenes.org/releases/19.html.

29. Li R, Qian J, Wang YY, Zhang JX, You YP. Long noncoding RNA profiles reveal three molecular subtypes in glioma. CNS Neurosci Ther. 2014; 20:339-43. https://doi. org/10.1111/cns.12220.

30. Zhang X, Sun S, Pu JK, Tsang AC, Lee D, Man VO, Lui WM, Wong ST, Leung GK. Long non-coding RNA expression profiles predict clinical phenotypes in glioma. Neurobiol Dis. 2012; 48:1-8. https://doi.org/10.1016/j. nbd.2012.06.004.

31. Zhang XQ, Kiang KM, Wang YC, Pu JK, Ho A, Cheng SY, Lee D, Zhang PD, Chen JJ, Lui WM, Fung CF, Leung GK. IDH1 mutation-associated long non-coding RNA expression profile changes in glioma. J Neurooncol. 2015; 125:253-63. https://doi.org/10.1007/s11060-015-1916-9.

32. Zhang XQ, Sun S, Lam KF, Kiang KM, Pu JK, Ho AS, Lui WM, Fung CF, Wong TS, Leung GK. A long noncoding RNA signature in glioblastoma multiforme predicts survival. Neurobiol Dis. 2013; 58:123-31. https://doi. org/10.1016/j.nbd.2013.05.011.

33. Chen Y, Wu JJ, Lin XB, Bao Y, Chen ZH, Zhang CR, Cai Z, Zhou JY, Ding MH, Wu XJ, Sun W, Qian J, Zhang L, et al. Differential lncRNA expression profiles in recurrent gliomas compared with primary gliomas identified by microarray analysis. Int J Clin Exp Med. 2015; 8:5033-43.

34. Yan Y, Zhang L, Jiang Y, Xu T, Mei Q, Wang H, Qin R, Zou Y, Hu G, Chen J, Lu Y. LncRNA and mRNA interaction study based on transcriptome profiles reveals potential core genes in the pathogenesis of human glioblastoma multiforme. J Cancer Res Clin Oncol. 2015; 141:827-38. https://doi.org/10.1007/s00432-014-1861-6.

35. Wang Y, Wang Y, Li J, Zhang Y, Yin H, Han B. CRNDE, a long-noncoding RNA, promotes glioma cell growth and invasion through mTOR signaling. Cancer Lett. 2015; 367:122-8. https://doi.org/10.1016/j.canlet.2015.03.027.

36. Zhang X, Rice K, Wang Y, Chen W, Zhong Y, Nakayama Y, Zhou Y, Klibanski A. Maternally expressed gene 3 (MEG3) noncoding ribonucleic acid: isoform structure, expression, and functions. Endocrinology. 2010; 151:939-47. https:// doi.org/10.1210/en.2009-0657.

37. Guo X, Gao L, Liao Q, Xiao H, Ma X, Yang X, Luo H, Zhao G, Bu D, Jiao F, Shao Q, Chen R, Zhao Y. Long non-coding RNAs function annotation: a global prediction method based on bi-colored networks. Nucleic Acids Res. 2013; 41:e35. https://doi.org/10.1093/nar/gks967.

38. Di Ruscio A, Ebralidze AK, Benoukraf T, Amabile G, GoffLA, Terragni J, Figueroa ME, De Figueiredo Pontes LL, Alberich-Jorda M, Zhang P, Wu M, D'Alo F, Melnick 
A, et al. DNMT1-interacting RNAs block gene-specific DNA methylation. Nature. 2013; 503:371-6. https://doi. org/10.1038/nature12598.

39. Keniry A, Oxley D, Monnier P, Kyba M, Dandolo L, Smits G, Reik W. The H19 lincRNA is a developmental reservoir of miR-675 that suppresses growth and Igflr. Nat Cell Biol. 2012; 14:659-65. https://doi.org/10.1038/ncb2521.

40. Ernst A, Campos B, Meier J, Devens F, Liesenberg F, Wolter M, Reifenberger G, Herold-Mende C, Lichter P, Radlwimmer B. De-repression of CTGF via the miR-1792 cluster upon differentiation of human glioblastoma spheroid cultures. Oncogene. 2010; 29:3411-22. https:// doi.org/10.1038/onc.2010.83.

41. Poliseno L, Salmena L, Zhang J, Carver B, Haveman WJ, Pandolfi PP. A coding-independent function of gene and pseudogene mRNAs regulates tumour biology. Nature. 2010; 465:1033-8. https://doi.org/10.1038/nature09144.

42. Kim K, Jutooru I, Chadalapaka G, Johnson G, Frank J, Burghardt R, Kim S, Safe S. HOTAIR is a negative prognostic factor and exhibits pro-oncogenic activity in pancreatic cancer. Oncogene. 2013; 32:1616-25. https://doi. org/10.1038/onc.2012.193.

43. Zhang L, Yang F, Yuan JH, Yuan SX, Zhou WP, Huo XS, $\mathrm{Xu}$ D, Bi HS, Wang F, Sun SH. Epigenetic activation of the MiR-200 family contributes to H19-mediated metastasis suppression in hepatocellular carcinoma. Carcinogenesis. 2013; 34:577-86. https://doi.org/10.1093/carcin/bgs381.

44. Torres-Garcia W, Zheng S, Sivachenko A, Vegesna R, Wang Q, Yao R, Berger MF, Weinstein JN, Getz G,
Verhaak RG. PRADA: pipeline for RNA sequencing data analysis. Bioinformatics. 2014; 30:2224-6. https://doi. org/10.1093/bioinformatics/btu169.

45. http://broadinstitute.github.io/picard/.

46. Anders S, Pyl PT, Huber W. HTSeq - a Python framework to work with high-throughput sequencing data. Bioinformatics. 2015; 31:166-9. https://doi.org/10.1093/bioinformatics/btu638.

47. Love MI, Huber W, Anders S. Moderated estimation of fold change and dispersion for RNA-seq data with DESeq2. Genome Biol. 2014; 15:550. https://doi.org/10.1186/ s13059-014-0550-8.

48. Griffiths-Jones S. The microRNA Registry. Nucleic Acids Res. 2004; 32:D109-11. https://doi.org/10.1093/nar/gkh023.

49. Ambros V, Bartel B, Bartel DP, Burge CB, Carrington JC, Chen X, Dreyfuss G, Eddy SR, Griffiths-Jones S, Marshall M, Matzke M, Ruvkun G, Tuschl T. A uniform system for microRNA annotation. RNA. 2003; 9:277-9.

50. Jeggari A, Marks DS, Larsson E. miRcode: a map of putative microRNA target sites in the long non-coding transcriptome. Bioinformatics. 2012; 28:2062-3. https:// doi.org/10.1093/bioinformatics/bts344.

51. Dweep H, Sticht C, Pandey P, Gretz N. miRWalk - database: prediction of possible miRNA binding sites by "walking" the genes of three genomes. J Biomed Inform. 2011; 44:839-47. https://doi.org/10.1016/j.jbi.2011.05.002. 